\title{
MODELADO DE LA FUENTE SÍSMICA DEL SISMO DE TECOMÁN DEL 21 DE ENERO DE 2003 UTILIZANDO EL MÉTODO DE LAS FUNCIONES DE GREEN EMPÍRICAS.
}

\author{
Alejandro Ramírez Gaytán ${ }^{(1)}$ y Jorge Aguirre González ${ }^{(1)}$.
}

\begin{abstract}
RESUMEN
Haciendo uso del método de las funciones de Green empíricas, de los registros de los sismos del 21 de enero de $2003\left(M_{w} 7.5\right)$ y 19 de noviembre de $2006\left(M_{w} 5.5\right)$, se generan modelos de fuente con diferente número, distribución y tamaño de las áreas de generación de movimientos fuertes SMGA (Strong Motion Generation Area) para el sismo de Tecomán. Estos modelos son probados utilizando cuatro estaciones regionales y una cercana a la fuente. Los modelos que se presentan corresponden a aquellos que mostraron los mejores ajustes en aceleración, velocidad y desplazamiento con base en los registros observados y los sintéticos. El modelo que mejor reproduce los registros observados se compone de tres SMGA, sus localizaciones parecen estar acorde con el modelo de dislocación encontrado en trabajos previos. La aplicación de modelos de fuente como el aquí desarrollado permitirá conocer las historias de aceleración, espectros de respuesta, aceleraciones máximas PGA (Peak Ground Acceleration) e Intensidades de Mercalli Modificadas $\left(\mathrm{I}_{\mathrm{MM}}\right)$ del sismo de Tecomán en lugares donde no existía instrumentación en el momento del sismo. Este trabajo aporta elementos originales y poderosos de la sismología que permiten generar aplicaciones útiles a la ingeniería sísmica en nuestro país.
\end{abstract}

\begin{abstract}
Using the empirical Green functions method, the records of January 21, 2003 (Mw 7.5) and November 19, 2006 (Mw 5.5) earthquakes, source models are generated with different numbers, distribution and size of Strong Motion Generation Area (SMGA) for the Tecomán earthquake. These models are tested using four regional stations and one station close to the source. The models presented in this paper correspond to those that showed the best fittings in acceleration, velocity and displacement, based on the observed and synthetic records. The model that best reproduces the observed records consists of three SMGA and their location appears to be consistent with the dislocation models found by others authors. The application of source models as developed in this study will allow in the future estimating the acceleration histories, the response spectra, Peak Ground Acceleration (PGA) and Modified Mercalli Intensities ( $\mathrm{I}_{\mathrm{MM}}$ ) for Tecomán earthquake in zones where no instrumentation existed at the time of the earthquake. The present work here developed provides powerful and original elements from the seismology that allow us to generate new and useful applications to seismic engineering in our country.
\end{abstract}

\footnotetext{
Artículo recibido el 14 de noviembre de 2007 y aprobado para su publicación el 14 de julio de 2008. Se aceptarán comentarios y/o discusiones hasta cinco meses después de su publicación

1 Instituto de Ingeniería, Universidad Nacional Autónoma de México, Circuito Interior, Ciudad Universitaria, Del. Coyoacán, México 04510, D.F., gramirezg@iingen.unam.mx, joagg@pumas.iingen.unam.mx
} 


\section{INTRODUCCIÓN}

Colima es uno de los estados de México que está sujeto a la ocurrencia de sismos destructivos. Buena parte de esta sismicidad se origina en la zona de subducción, a lo largo de la costa del Pacífico mexicano. Si bien la tasa de sismicidad es menor a la observada en el estado de Guerrero, ésta no es despreciable. Ejemplos de esto son los sismos de $1932\left(\mathrm{M}_{\mathrm{w}}\right.$ 8.2) y de Tecomán del 21 de enero de $2003\left(M_{w}\right.$ 7.5), ver figura 1, que son unos de los sismos de mayor magnitud registrados en todo el territorio nacional durante el siglo pasado y el presente, respectivamente. (Cabe aclarar que el sismo del 19 de septiembre de 1985 tuvo una magnitud $\mathrm{M}_{\mathrm{w}} 8.0$ y el de Manzanillo del 9 de octubre de 1995 una magnitud $\mathrm{M}_{\mathrm{w}} 8.0$, fuente CMT de Harvard).

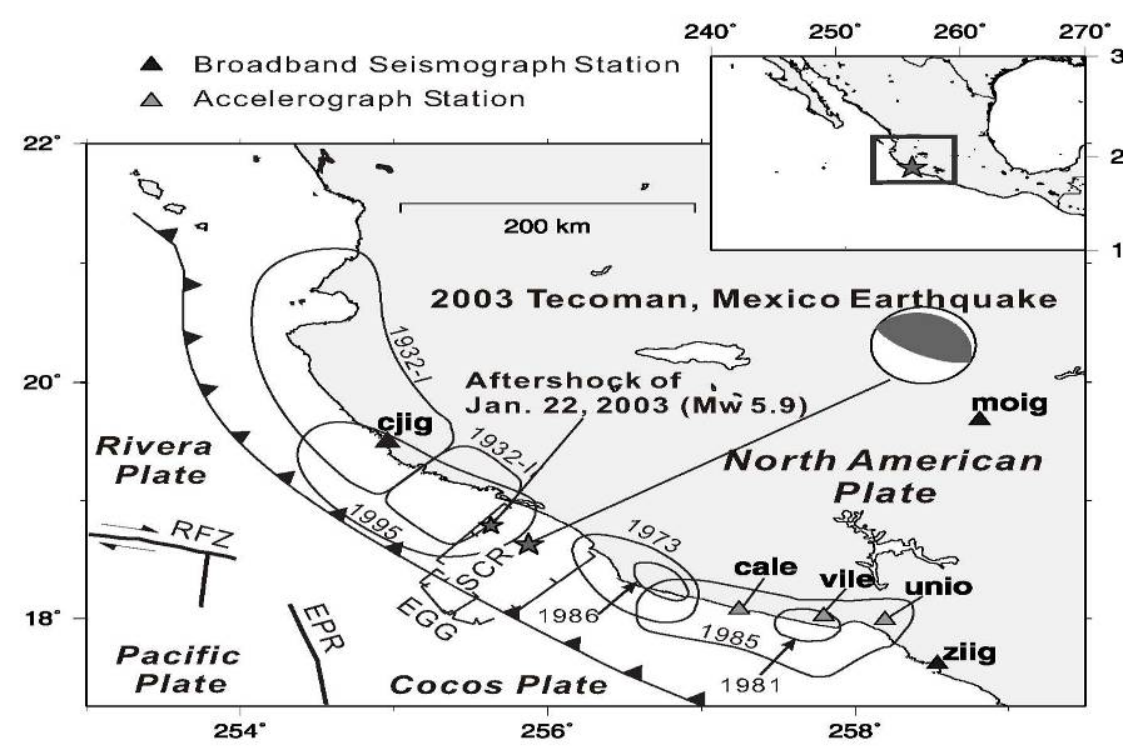

Figura 1. Mapa tectónico de la región (tomado de Yagi et al., 2004). Estrella grande: Sismo de

Tecomán, estrellas pequeñas: réplicas, RFZ: Zona de fractura de la placa de Rivera (Rivera

Fracture Zone), SCR: Rift del sur de Colima (Sourthern Colima Rift), EGG: Graben el

Gordo. Los contornos muestran el área de réplicas de los grandes sismos de la región. Los triángulos negros indican las estaciones de banda ancha que registraron el sismo.

Aunque se han realizado un buen número de investigaciones sobre los grandes sismos que han afectado la región, éstas se han realizado con base en registros telesísmicos y regionales. Sin embargo, la ausencia de estos registros en sitios cercanos al epicentro y por ende de investigaciones que utilicen este tipo de datos, demuestra que la instrumentación y la sismicidad en Colima no habían recibido una atención adecuada hasta el año 2005.

El principal objetivo de este artículo es abordar el estudio de la fuente sísmica en la región utilizando datos en sitios cercanos al epicentro, lo que hasta la fecha no se había realizado. Este objetivo se vió limitado en un principio, debido a que hasta el año 2005 Colima contaba con una sola estación de aceleración. Aunque ya existía la Red Sísmica del estado de Colima (RESCO), las características de esta red, cuyo fin es monitorear la actividad del volcán de Colima, no permitieron obtener registros sísmicos útiles, ya que éstos se saturaron durante el sismo de Tecomán. Para revertir esta situación se inició un proyecto que tuvo entre sus principales objetivos instrumentar permanentemente la región. Para este fin, se instaló una red temporal compuesta por 16 acelerógrafos y 9 sismógrafos de banda corta, tanto en el interior del estado como a lo largo de los $120 \mathrm{~km}$ de costa con que cuenta. 
Como producto del trabajo de instrumentación mencionado, el 19 de noviembre de 2006 se logró registrar el evento de mayor importancia desde el sismo de 2003; su epicentro se observa en la figura 2. Sus características (localización y mecanismo focal) aparecen en la tabla 1. Como se puede observar, tanto su ubicación como su mecanismo focal coinciden con los del sismo del 21 de enero de 2003 (sismo de Tecomán), por lo que ambos sismos pertenecen al mismo ambiente tectónico. De hecho el epicentro del sismo del 19 de noviembre de 2006 se encuentra dentro del área de ruptura reportada por Yagi et al. (2004) para el sismo de Tecomán.

Tabla 1. Datos de localización del sismo principal y del sismo utilizado como función de Green empírica.

\begin{tabular}{lcc}
\hline \multicolumn{1}{c}{ Parámetro del sismo } & Sismo principal & EGF evento \\
\hline Tiempo origen & $21 / 03 / 200320: 06$ & $19 / 11 / 200606: 59$ \\
Localización hipocentral: latitud, & $\left(18.60^{\circ} \mathrm{N}, 104.22^{\circ} \mathrm{W}, 26 \mathrm{Km}\right)$ & $\left(18.46^{\circ} \mathrm{N}, 104.49^{\circ} \mathrm{W}, 18 \mathrm{~km}\right)$ \\
longitud y profundidad (fuente SSN $)$ & $7.5 \mathrm{M}_{\mathrm{w}}$ & $5.5 \mathrm{M}_{\mathrm{w}}$ \\
$\begin{array}{l}\text { Magnitud } \\
\text { Mecanismo focal: acimut, echado, }\end{array}$ & $\left(308^{\circ}, 12^{\circ}, 110^{\circ}\right)$ & $\left(305^{\circ}, 23^{\circ}, 82^{\circ}\right)$ \\
$\begin{array}{l}\text { ángulo de deslizamiento (fuente } \\
\text { CMT de Harvard) }\end{array}$ & & \\
\hline
\end{tabular}

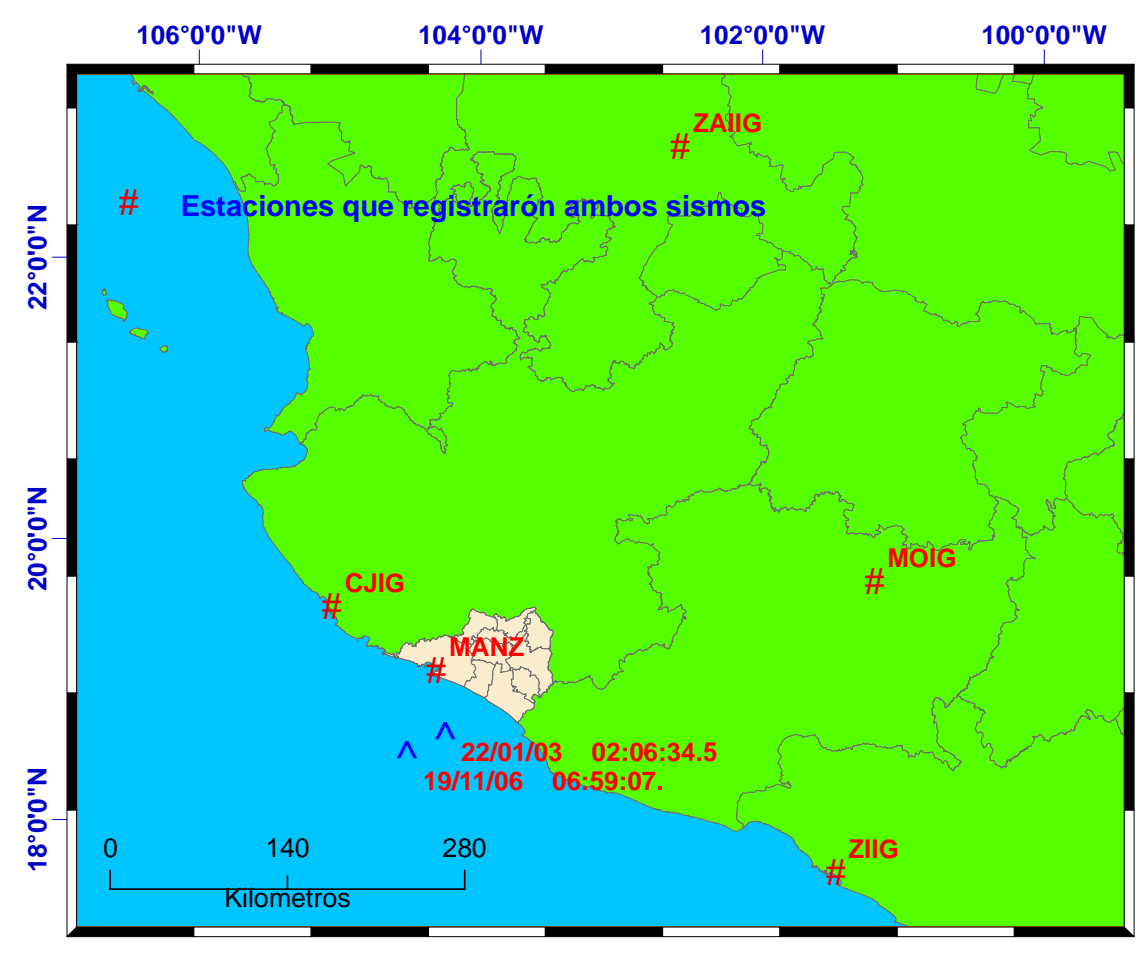

Figura 2. Las cinco estaciones que registraron tanto el terremoto de Tecomán como el sismo del 19 de noviembre de 2006.

Además de ser registrado por esta red, dicho temblor fue registrado por la estación de aceleración local en Manzanillo (MANZ) y por 4 estaciones de velocidad del Servicio Sismológico Nacional (SSN), mismas que registraron el sismo de Tecomán.

Las características mencionadas con anterioridad permiten aplicar al sismo del 19 de noviembre de 2006 el método de las funciones de Green empíricas y de esta manera escalarlo y reproducir el 
sismo de Tecomán (tabla 1). Con este método se estimaron las áreas de generación de movimientos fuertes (SMGA, Strong Motion Generation Area), utilizando la banda de frecuencias entre 1.0 y 10 $\mathrm{Hz}$, altas frecuencias que no pueden ser modeladas por otras aproximaciones teóricas debido a la falta de información de la estructura cortical. Las SMGAs son áreas rectangulares en las que el desplazamiento excede al deslizamiento promedio en la falla; se considera que en esta zona el deslizamiento, la caída de esfuerzos y la velocidad de ruptura son constantes (Miyake et al., 2003). Este modelo de fuente ha sido exitosamente aplicado para simular los registros de aceleración de algunos sismos grandes y moderados (Nozu e Irikura, 1998;Kamae e Irikura, 2008 y Aguirre et al. ,1994).

A diferencia de las SMGAs, las asperezas son estimadas por medio de inversiones que utilizan bajas frecuencias en el rango de $0.1 \mathrm{a} 0.5 \mathrm{~Hz}$. Una aspereza se define como una región dentro del área de dislocación en la cual el deslizamiento excede al deslizamiento promedio en toda el área de ruptura (Somerville et al., 1999). Miyake et al. (2003) muestran que las SMGAs se localizan casi en las mismas posiciones que las asperezas y que éstas corresponden aproximadamente a las áreas de máxima dislocación de acuerdo al criterio establecido por Somerville et al. (1999).

Con base en lo anterior, las SMGAs del mejor modelo encontrado se compararon con el modelo de dislocación encontrado por Yagi et al. (2004). Somerville et al. (1999) proponen que la dislocación para sismos de subducción se caracterice mediante una variación espacial de las asperezas en la superficie de falla y examinan cómo los modelos de dislocación se escalan con el momento sísmico. Dado que las SMGAs que se obtuvieron corresponden, aproximadamente, a las áreas de máxima dislocación, se cuantificaron sus características de manera individual y en conjunto. Al compararlas con las relaciones propuestas por Somerville et al. (2002), se discute la validez de su aplicación a la zona de subducción en estudio.

\section{TECTÓNICA Y SISMICIDAD HISTÓRICA}

Son tres las fuentes sísmicas en el estado de Colima: la primera de ellas, el volcán de Colima que genera microtremores y sismos con bajas magnitudes generalmente menores a 3.5. La segunda fuente sísmica proviene del bloque de Jalisco, que se encuentra en la placa Norteamericana y limita hacia el éste con la placa de Rivera, hacia el sur con el graben de Colima y el graben El Gordo (EGG por sus siglas en inglés) y en el norte con el graben de Tepic y el graben de Chapala (Bandy et al., 1995). En esta región han ocurrido sismos intraplaca de importancia con magnitudes no mayores a 6.0. En la red de acelerógrafos que se comenta en este artículo, se logró registrar una actividad sísmica importante que parece cortarse precisamente en los límites del graben El Gordo con el bloque de Jalisco. Sin embargo, la fuente sísmica más importante en la región es la zona de subducción frente a las costas del estado de Colima, en donde convergen las placas de Rivera, de Cocos y de Norteamérica. Las placas de Cocos y Rivera se subducen bajo la de Norteamérica a una velocidad promedio de $5 \mathrm{~cm}$ por año. Este mecanismo ocurre frente a las costas del estado en la zona del graben El Gordo, como puede apreciarse en la figura 1.

El sismo de Tecomán ocurrió en la brecha sísmica que forman los límites del área de ruptura de los sismos de 1973 y 1995. Sin embargo, la localización de las réplicas del sismo de Tecomán indican que su área de ruptura abarca la parte norte del graben El Gordo e invaden parcialmente la zona de ruptura de los sismos de 1932 y 1995. La ausencia de réplicas hacia el suroeste indica que la mitad de esta brecha sísmica no rompió.

Los sismos de 1995 y 1932, aunque tienen áreas de ruptura distintas, tienen el mismo momento sísmico $\left(\mathrm{Mo}=9.0 \times 10^{27}\right.$ dina-cm), lo cual pudo ser causado por la diferencia entre las cantidades de desplazamiento total en cada uno de ellos. Esto indica que el sismo de 1995 liberó una cantidad de energía equivalente a la del sismo más importante registrado en la historia del país. 
La gran diferencia entre el momento sísmico de estos dos sismos con el del sismo de Tecomán (2.05 x $10^{27}$ dina-cm), muestra que el sismo de Tecomán fue mucho menos energético que los sismos de 1995 y 1932. Sin embargo los daños causados por éste fueron mucho mayores en las zonas urbanas del estado con excepción de Manzanillo. La directividad es una de las causas que pueden explicar este fenómeno. La directividad del sismo hacia el interior de la placa Norteamericana coincide con la ubicación geográfica de 8 de las 10 principales zonas urbanas del estado. Ya que estas zonas urbanas, junto con la zona de ruptura del sismo de Tecomán se encuentran dentro del graben de Colima, es de importancia en futuros proyectos estudiar el papel que juega este graben en la conducción de la energía sísmica (transmisión, reflexión y refracción de ondas) producida por esta fuente. Además deben considerarse los efectos de sitio producidos por el tipo de depósitos aluviales en el graben. Estos dos fenómenos en conjunto pueden ser causantes de grandes aceleraciones en la zona. La diferencia entre los momentos sísmicos de los sismos de 1995 y 2003, que comparten una zona del área de ruptura, sugiere que si se generara un sismo de magnitud similar a la del sismo de 1995 en la parte de la brecha sísmica ubicada dentro del graben de Colima, se generaría un escenario sísmico muy crítico para la región.

\section{DATOS}

Se recolectaron los registros para el sismo principal del 21 de enero de 2003 (sismo de Tecomán) y para el sismo secundario del 19 de noviembre de 2006, ambos de la estación de aceleración (MANZ), que pertenece a la Comisión Federal de Electricidad y es operada por el Centro de Instrumentación y Registro Sísmico (CIRES). Esta estación se localiza a $54 \mathrm{~km}$ del epicentro y es la estación de aceleración más cercana al epicentro que registró el sismo de Tecomán. Está equipada con un acelerógrafo modelo DCA-333 de tres componentes, que registra con una velocidad de muestreo de 100 muestras por segundo, y tiene un umbral de disparo de 4.9 gals.

Se intentó recolectar los registros de aceleración para ambos eventos de las estaciones de Chamela (CJIG), Morelia (MOIG), Zacatecas (ZAIG), y Zihuatanejo (ZIIG), pertenecientes al Servicio Sismológico Nacional (SSN), que cuentan con una buena cobertura acimutal alrededor de la zona. Sin embargo, de acuerdo con la información proporcionada por personal de SSN, los registros de aceleración de esa época no fueron almacenados, razón por la que se tuvo que trabajar con los registros de velocidad. Los cuatro registros de velocidad fueron registrados en sismógrafos de banda ancha. Los equipos de las estaciones CJIG y ZIIG tienen una velocidad de muestreo de 80 muestras por segundo. Las estaciones MOIG y ZAIG tienen una velocidad de muestreo de 100 muestras por segundo. Los datos fueron cambiados del formato proporcionado a formato ASCII y posteriormente transformados a aceleración.

Las complejidades en el proceso de conversión surgen cuando se convierten aceleraciones a velocidades, en este caso es necesario realizar una corrección por línea base y un filtrado para evitar la acumulación de errores por el proceso de integración numérica. El proceso de convertir registros de velocidad a aceleración, presenta menos complejidades debido a que involucra una derivación numérica. No obstante después de realizar la derivación numérica los registros fueron corregidos por línea base. De este proceso resultan incertidumbres que consideramos mínimas cuya influencia es prácticamente despreciable.

El mecanismo focal adoptado para el sismo principal fue el reportado por el CMT de Harvard: acimut $308^{\circ}$, echado $12^{\circ}$, ángulo de deslizamiento $110^{\circ}$. De igual forma, el mecanismo focal adoptado para el sismo pequeño correspondió al reportado por el CMT de Harvard: acimut $305^{\circ}$, echado $23^{\circ}$, ángulo de deslizamiento $82^{\circ}$, (tabla 1). El área de dislocación asumida es la reportada por Yagi et al. (2004) de 70 x $85 \mathrm{~km}$. 
A partir de las señales acelerográficas, se obtuvieron los niveles planos de los espectros de desplazamiento para las bajas frecuencias, y los niveles planos de los espectros de aceleración para las altas frecuencias en la estación MANZ. Con base en el análisis de estos espectros, se encontró que los niveles planos para los espectros de aceleración se localizan entre 1.0 y $10 \mathrm{~Hz}$; además, se obtuvieron las frecuencias de esquina $(\mathrm{Fc})$ del sismo principal y del sismo pequeño.

\section{MÉTODO}

El método aplicado requiere de un sismo de menor magnitud (sismo del 11 de noviembre de 2006) cercano al sismo principal para poder modelar el de mayor magnitud (sismo de Tecomán del 21 de enero de 2003). Con el método sintético para el modelo espectral $\omega^{-2}$ propuesto por Aki (1967), se obtiene el número de subeventos necesarios $N^{3}$, a partir de la relación entre los momentos sísmicos del sismo principal que será simulado y el de la réplica que se usa como función de Green, donde $N^{3}$ es igual al número de subfallas en dirección del rumbo $\left(N_{x}\right)$, del echado $\left(N_{w}\right)$ y del tiempo $\left(N_{t}\right)$,

$$
\mathrm{N}^{3}=\mathrm{N}_{\mathrm{x}} \times \mathrm{N}_{\mathrm{w}} \times \mathrm{N}_{\mathrm{t}}
$$

Se debe encontrar entonces el parámetro $N$ que sirve para estimar el área de la falla del sismo principal. Ya que se divide en $N X N$ subfallas, el cual se obtiene de la siguiente manera:

$$
\frac{\bar{U}_{0}}{\bar{u}_{0}}=\frac{M_{0}}{m_{0}}=N^{3} \text {. }
$$

donde: $\bar{U}$ y $\bar{u}$ es el nivel plano de los espectros de Fourier de los registros de desplazamiento para el sismo principal y el sismo pequeño respectivamente. Mo y mo son los momentos sísmicos del movimiento principal y del sismo pequeño respectivamente. La relación para las altas frecuencias esta dado por:

$$
\frac{\overline{A_{0}}}{\bar{a}_{0}}=\left(\begin{array}{c}
M_{0} \\
m_{0}
\end{array}\right)^{1 / 3}=N .
$$

donde: $\bar{A}$ y $\bar{a}$ son el nivel plano de los espectros de Fourier de los registros de aceleración del sismo principal y del sismo pequeño, respectivamente. Entonces el movimiento sintético del sismo principal $A(t)$, estará dado por el movimiento observado del sismo pequeño $a(t)$, con base en las siguientes ecuaciones:

$$
\begin{aligned}
& A(t)=\sum_{i=1}^{N x} \sum_{j=1}^{N w}\left(\frac{r}{r_{i j}}\right) F\left(t-t_{i j}\right) * a(t) . \\
& F_{i j}\left(t-t_{i j}\right)=\delta\left(t^{\prime}-t_{i j}\right)+\frac{1}{n^{\prime}} \sum_{k=1}^{(N-1) n^{\prime}} \delta\left[t-t_{i j}-\frac{(k-1) \tau}{(N-1) n^{\prime}}\right] .
\end{aligned}
$$

donde $n$ ' es un entero apropiado para eliminar periodicidad espuria, $r$ es la distancia de la estación al hipocentro del sismo pequeño, $r_{i j}$ es la distancia de la estación al elemento $(i, j), t_{i j}$ es el tiempo de retraso para la ruptura del punto de salida del elemento $(i, j)$ al sitio observado, $\tau$ es el tiempo de ascenso (rise time). Para usar el movimiento de un sismo pequeño con caída de esfuerzos diferente a la del sismo principal, se modifica la función de Green empírica, introduciendo un valor constante que sirve para corregir la diferencia entre la caída de esfuerzos del sismo principal y de la réplica, tal que: 


$$
C=\frac{\Delta \sigma_{S P}}{\Delta \sigma_{R}} .
$$

donde $\Delta \sigma_{S P}$ es la caída de esfuerzos del sismo principal y $\Delta \sigma_{R}$ es la caída de esfuerzos de la réplica. El nivel espectral es afectado por el mismo factor de la ecuación anterior, por lo que queda de la siguiente manera:

$\overline{\mathrm{U}}_{0} / \overline{\mathrm{u}}_{0}=\mathrm{CN}^{\prime 3}$.

$\overline{\mathrm{A}} / \overline{\mathrm{a}}=\mathrm{CN}^{\prime}$.

La ecuación 4 se modifica reemplazando $a(t) \operatorname{con} C a(t)$ y $N \operatorname{con} N^{\prime}$ como sigue ( Irikura, 1986):

$$
\begin{aligned}
& A(t)=\sum_{i=1}^{N^{\prime}} \sum_{j=1}^{N^{\prime}}\left(\frac{r}{r_{i j}}\right) F\left(t-t_{i j}\right) * C a(t) . \\
& F_{i j}\left(t-t_{i j}\right)=\delta\left(t-t_{i j}\right)+\frac{1}{n^{\prime}} \sum_{k=1}^{\left(N^{\prime}-1\right) n^{\prime}} \delta\left[t-t_{i j}-\frac{(k-1) \tau}{\left(N^{\prime}-1\right) n^{\prime}}\right] .
\end{aligned}
$$

\section{MODELO}

Para el modelo del evento principal, se asume un área de dislocación de 70 x $85 \mathrm{~km}$ (Reportada por Yagi et al., 2004). Se observa que el mejor modelo encontrado divide la SMGA en tres zonas, una primera zona localizada a $20.75 \mathrm{~km}$ al SW del epicentro, una segunda zona a $10.24 \mathrm{~km}$ al SE del epicentro y la tercera a $20.16 \mathrm{~km}$ al NE del epicentro. Debido a que la caída de esfuerzos es distinta para el evento principal y para el evento pequeño, se aplicó un método apropiado (ecuaciones 4 a 10). De esta forma se obtuvo el parámetro $N=8$ y $C=1.08$. Además, se necesitó asumir y variar otros parámetros para simular los registros de aceleración del sismo principal, tales como la velocidad de ruptura, tiempo de dislocación y punto en que inicia la ruptura. Aplicando este modelado directo, se observa poca sensibilidad de los registros sintéticos al variar el tiempo de ascenso (rise time), en cambio, una alta sensibilidad al variar la velocidad de ruptura, el tamaño de las SMGAs y su localización dentro del plano de falla. Aplicando el método, se generó una gran cantidad de modelos en que se variaron los parámetros mencionados. El mejor modelo se determinó minimizando los residuales entre los sintéticos y los observados.

\section{RESULTADOS}

El criterio para determinar cuál es el modelo más cercano a la fuente real se basa en encontrar el mejor ajuste entre la traza sintética y la observada. Encontrar este mejor ajuste consiste en generar modelos en los que se varían diversos parámetros de la fuente. El modelo que generó un sintético lo más cercano al observado en las cinco estaciones utilizadas, es precisamente el que se toma como modelo de la fuente. Esto significa que el ajuste de los registros depende directamente de los parámetros de la fuente considerados.

Que un modelo obtenido reprodujera adecuadamente la traza observada en una estación no fue suficiente para considerarlo el modelo de fuente para el sismo de Tecomán, este mismo modelo debería de reproducir adecuadamente cualquiera de las trazas observadas en las otras cuatro estaciones. Por esta razón, se entra en un proceso cíclico de modelado basado en la optimización y discriminación que requirió más de 160 interacciones. En cada una de éstas se varió la distribución, tamaño y cantidad de las SMGAs dentro del área de dislocación, velocidad de ruptura, tiempo de 
ascenso (rise time) y tamaño de las subfallas. En algunos de los modelos probados se considero la corrección por patrón de radiación, aunque la influencia de éste en altas frecuencias es prácticamente despreciable. En otros casos se ajustó el tamaño de las subfallas de las SMGAs tanto en la dirección del acimut o del echado, según el acimut de la estación o estaciones que presentaron poco ajuste. Finalmente, en otros casos más se varió la localización de las SMGAs, acercándolas al foco e incrementando su velocidad de ruptura, tratando de compactar algunos paquetes de ondas dentro de la traza o viceversa.

El parámetro que mayor peso tuvo en el proceso del modelado fue la posición, el tamaño y el número de las SMGAs. Por esta razón el proceso ya descrito arriba generó tres etapas en las que la distribución y tamaño de las SMGAs fue variando dentro del área de dislocación acorde con el ajuste del sintético obtenido.
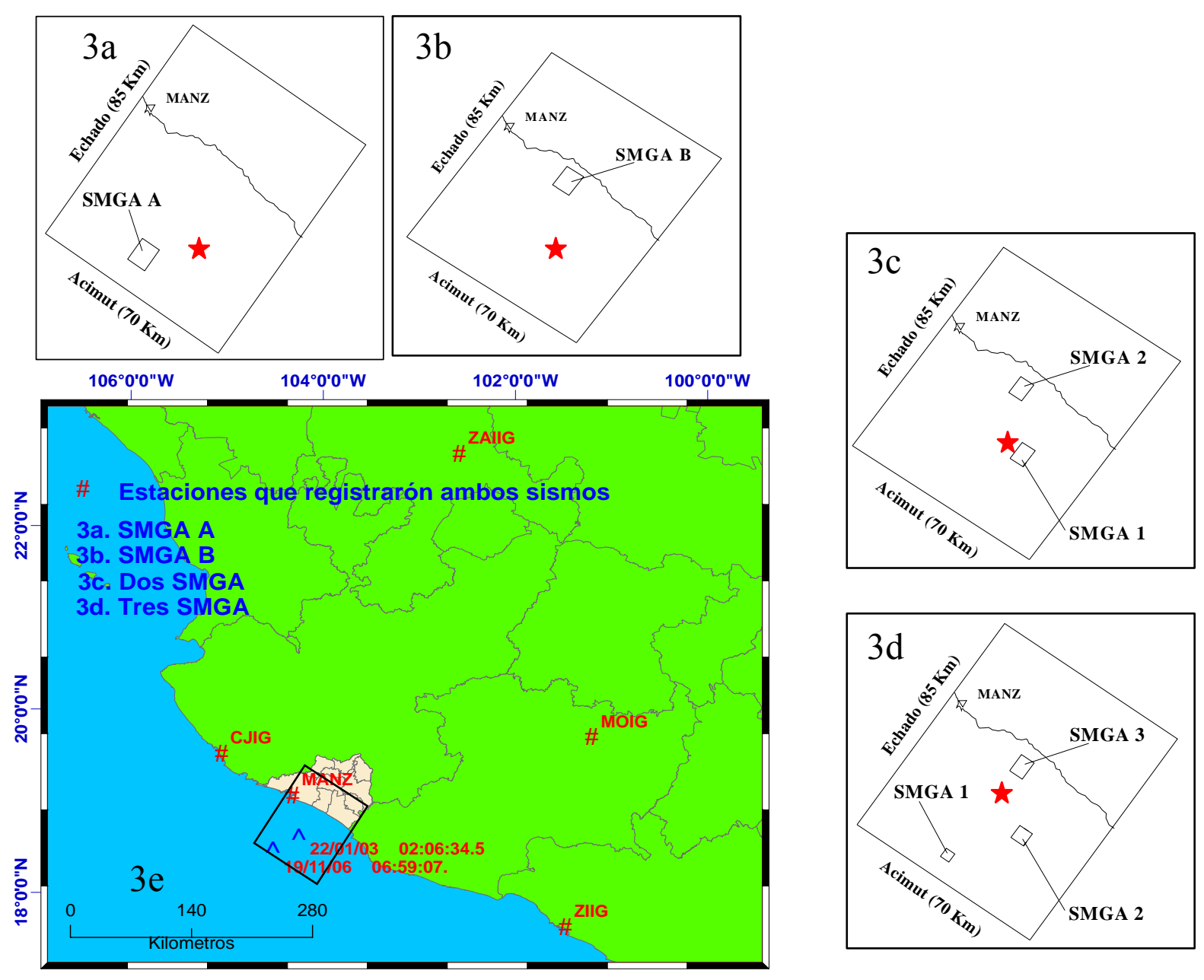

Figura 3. En 3a y $3 b$ los mejores modelos obtenidos con una SMGA; 3c y 3d mejor modelo obtenido con dos y tres SMGA respectivamente; 3e estaciones utilizadas en la modelación.

Para evaluar cuantitativamente el ajuste entre la señal sintética y la observada se utiliza el residual de acuerdo a la siguiente ecuación: 
residual $=\frac{\sum_{i=1}^{n}\left(\text { simulado }_{i}-\text { observado }_{i}\right)^{2}}{\sqrt{\sum_{i=1}^{n}\left(\text { simulado }_{i}{ }^{2}\right) \sqrt{\sum_{i=1}^{n}\left(\text { observado }_{i}{ }^{2}\right)}}}$

donde: $\operatorname{simulado}_{i}$ es la muestra i-ésima del registro sintético; observado $\mathrm{i}_{\mathrm{i}}$ es la muestra i-ésima del registro observado y $n$ es el número total de muestras consideradas para obtener el residual. En este caso $n$ varía de acuerdo al registro de cada estación. Un perfecto ajuste entre lo simulado y lo observado representaría un residual de cero.

Con este residual la normalización se hace con respecto a la energía total de la traza en el intervalo. Esto resulta apropiado considerando el carácter altamente oscilatorio (altas frecuencias) de las ondas comparadas. Es claro que los valores bajos resultan en una mejor aproximación de la traza en todo el intervalo.

Para considerar el efecto que tiene el modelo en forma global, se considera un promedio simple de las tres componentes en todas las estaciones, referido como residual promedio.

Los parámetros y pruebas mencionadas anteriormente se aplicaron en cada una de las tres etapas del proceso. En la primera etapa se modeló utilizando 1 SMGA y ajustándola a la estación más cercana a la fuente (MANZ). La localización de la SMGA se varió en todo el plano de falla. Los mejores modelos generados durante esta primera etapa se muestran al ubicar la única SMGA en las zonas señaladas como SMGA "A" (figura 3a) y SMGA "B" (figura 3b).

Tabla 2. Área y localización de las SMGAs para los cuatro modelos generados con una, dos y tres SMGA, respectivamente, donde Vr es la velocidad de ruptura.

\begin{tabular}{|c|c|c|c|c|c|c|}
\hline Modelo & SMGA & $\begin{array}{l}\text { Largo } \\
(\mathrm{km})\end{array}$ & $\begin{array}{l}\text { Ancho } \\
(\mathrm{km})\end{array}$ & $\begin{array}{c}\text { Área } \\
\left(\mathrm{km}^{2}\right)\end{array}$ & $\begin{array}{l}\text { Punto de inicio de la ruptura } \\
\text { con relación al epicentro }\end{array}$ & $\begin{array}{c}\mathrm{Vr} \\
\mathrm{km} / \mathrm{s}\end{array}$ \\
\hline 1 & 1 & 8.60 & 6.88 & 59.168 & $16.68 \mathrm{~km} \mathrm{SW}$ & 2.1 \\
\hline 2 & 1 & 8.60 & 6.88 & 59.168 & $21.27 \mathrm{~km} \mathrm{NE}$ & 2.1 \\
\hline \multirow{2}{*}{3} & 1 & 5.16 & 6.88 & $\begin{array}{r}35.500 \\
8\end{array}$ & $10.48 \mathrm{~km} \mathrm{SE}$ & 2.1 \\
\hline & 2 & 5.16 & 6.88 & $\begin{array}{r}35.500 \\
8\end{array}$ & $20.07 \mathrm{~km} \mathrm{NE}$ & 2.9 \\
\hline \multirow{3}{*}{4} & 1 & 3.44 & 3.44 & 11.83 & $20.75 \mathrm{~km} \mathrm{SW}$ & 2.1 \\
\hline & 2 & 5.16 & 5.16 & 26.62 & $10.24 \mathrm{~km} \mathrm{SE}$ & 2.9 \\
\hline & 3 & 6.88 & 5.16 & 35.50 & $20.16 \mathrm{~km} \mathrm{NE}$ & 2.1 \\
\hline
\end{tabular}

Tabla 3. Residuales en aceleración, velocidad y desplazamiento para el modelo con una SMGA

\begin{tabular}{|c|c|c|c|c|c|c|c|}
\hline & & \multicolumn{3}{|c|}{ Una SMGA (SMGA A) } & \multicolumn{3}{|c|}{ Una SMGA (SMGA B) } \\
\hline Est. & Componente & Acel. & Vel. & Desp. & Acel. & Vel. & Desp. \\
\hline \multirow{4}{*}{$\underset{\Sigma}{Z}$} & WE & 36.30 & 6.37 & 2.21 & 12.56 & 3.52 & 2.82 \\
\hline & SN & 61.81 & 14.92 & 5.41 & 5.61 & 6.07 & 2.63 \\
\hline & Vertical & 10.65 & 8.34 & 1.93 & 4.83 & 2.91 & 1.60 \\
\hline & $\sum$ & 108.75 & 29.62 & 9.55 & 23.00 & 12.50 & 7.05 \\
\hline
\end{tabular}

El primer mejor ajuste (SMGA "A") se obtuvo al modelar con una SMGA de $59.16 \mathrm{~km}^{2}$, $\mathrm{Vr}=2.1 \mathrm{~km} / \mathrm{s}$ (tabla 2), localizada a $16.68 \mathrm{~km}$ al SW del hipocentro. Los valores comparativos de residual para cada una las componentes en aceleración, velocidad y desplazamiento para ambos modelos, que aparecen en la tabla 3, muestran un mejor ajuste en las tres componentes al modelar con 
una SMGA (SMGA B) de $59.16 \mathrm{~km}^{2}, \mathrm{Vr}=2.1 \mathrm{~km} / \mathrm{s}$ (tabla 3), y ubicándola a $21.27 \mathrm{~km}$ al NE del hipocentro en la posición que se muestra en la figura $3 a$ y $3 b$.

La figura 4 (SMGA “A") y la figura 5 (SMGA "B") muestran la comparación entre los registros observados (rojo) y sintéticos (azul) para las tres componentes, en aceleración, velocidad y desplazamiento, en esta primera etapa.

\section{3/22/01 02:06:34.5 MANZ SMGA A}
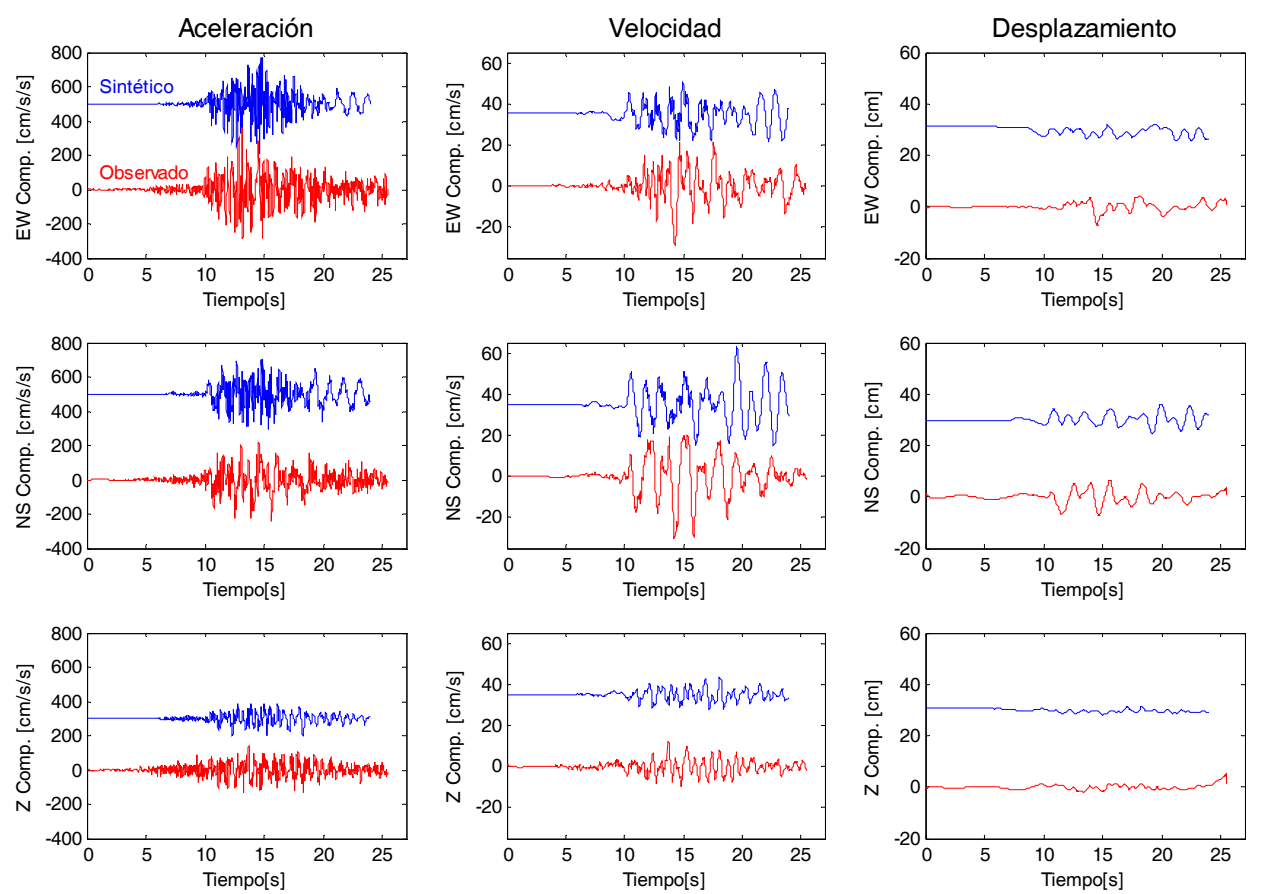

Figura 4. Simulación para la estación MANZ usando el modelo con una SMGA (SMGA A).

En la segunda etapa, se modeló con dos SMGA, ajustando los registros de la estación más cercana a la fuente (MANZ). La localización de estas dos SMGA se varió en todo el plano de falla. El modelo que arrojó el mejor ajuste se aplicó a los registros obtenidos de las estaciones regionales CJIG, MOIG, ZAIG y ZIIG, estaciones en que existen registros disponibles y que proporcionan una buena cobertura acimutal. Los valores de residual para las tres componentes en aceleración, velocidad y desplazamiento se presentan en la tabla 4 y muestran que el mejor ajuste se obtuvo para el modelo mostrado en la figura 3c. Este modelo consiste de dos SMGA; una de $35.50 \mathrm{~km}^{2}$ localizada a $10.48 \mathrm{~km}$ al SE del hipocentro y otra de $35.50 \mathrm{~km}^{2}$, localizada a $20.07 \mathrm{~km}$ al NE del epicentro, como se indica en la tabla 2. La figura 6, muestra la comparación entre los registros observados (rojo) y sintéticos (azul) para las tres componentes, en aceleración, velocidad y desplazamiento, de la estación MANZ.

En la tercera etapa se modeló con tres SMGA, ajustando los registros de la estación más cercana a la fuente (MANZ). La localización de estas tres SMGA se varió en todo el plano de falla. El modelo que arrojó el mejor ajuste también se aplicó a los registros obtenidos de las estaciones regionales CJIG, MOIG, ZAIG y ZIIG. Los valores de residual para las tres componentes en aceleración, velocidad y desplazamiento que aparecen en la tabla 4, muestran que el mejor ajuste se obtuvo para el modelo mostrado en la figura 3d. Este modelo consta de tres SMGA; una de $11.83 \mathrm{~km}^{2}$, localizada a $20.75 \mathrm{~km}$ al SW del epicentro, otra de $20.62 \mathrm{~km}^{2}$, localizada a $10.24 \mathrm{~km}$ al SE del epicentro y otra de $35.50 \mathrm{~km}^{2}$, localizada a $20.16 \mathrm{~km}$ al NE del hipocentro. Las características de este modelo se muestran en la tabla 2. 


\section{3/22/01 02:06:34.5 MANZ SMGA B}
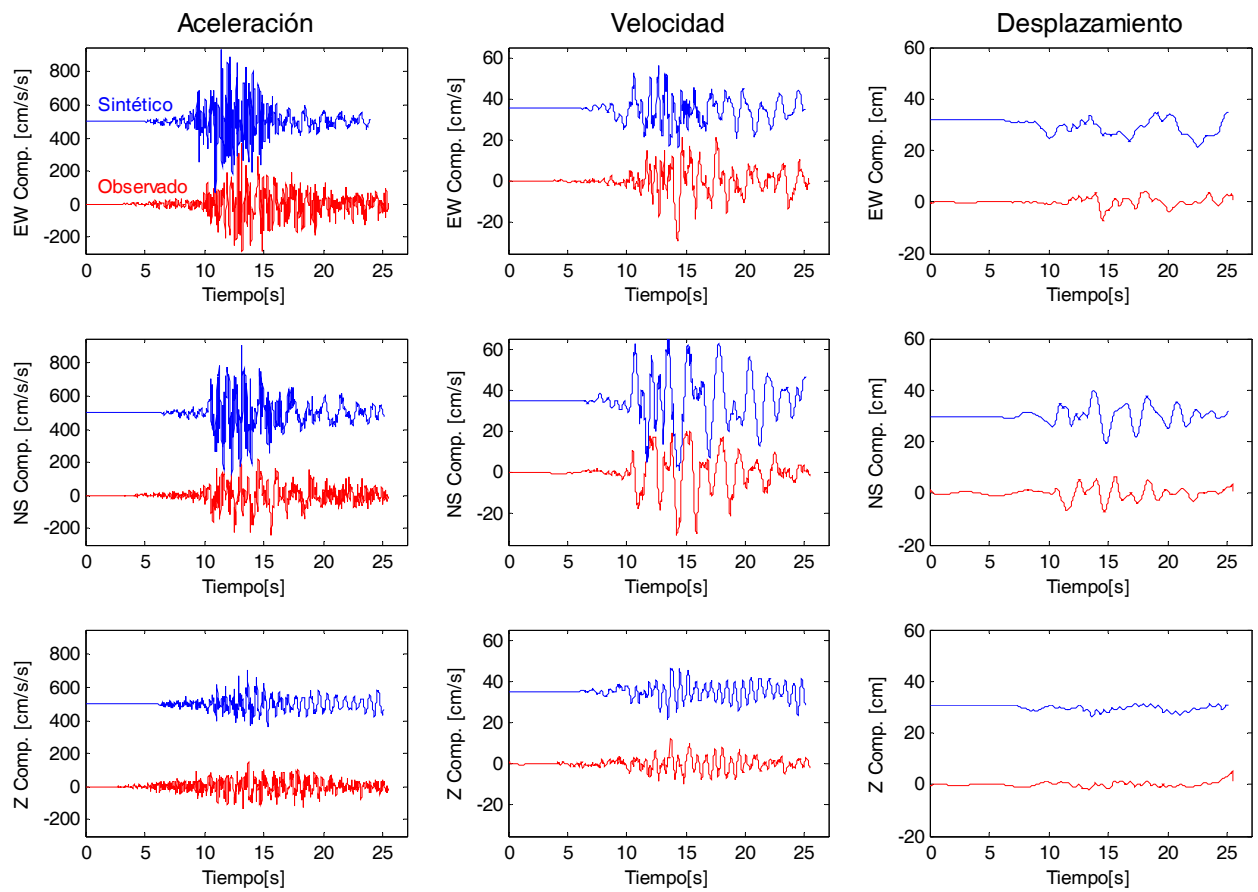

Figura 5. Simulación para la estación MANZ usando el modelo con una SMGA (SMGA B).

\section{3/22/01 02:06:34.5 MANZ (2 SGMA)}
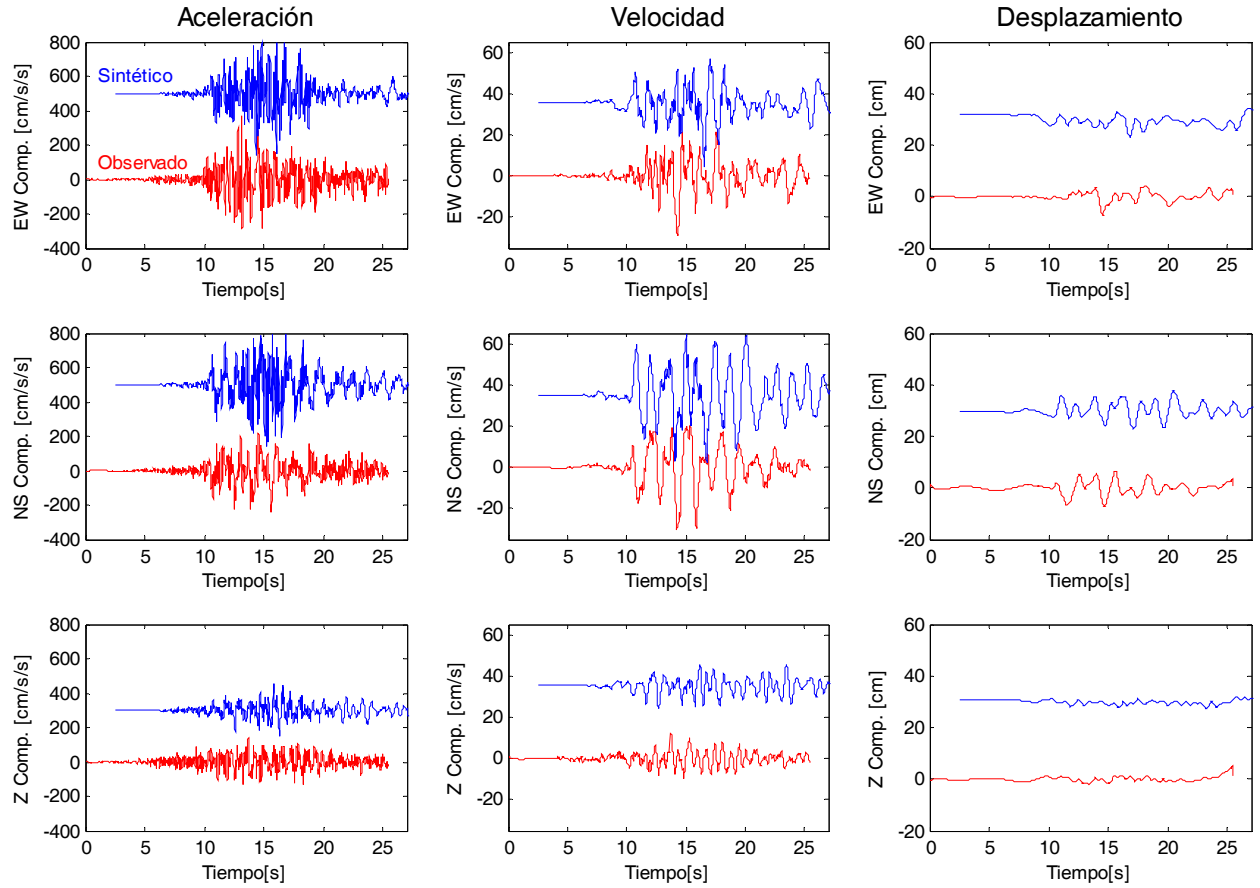

Figura 6. Simulación para la estación MANZ usando el modelo con dos SMGA. 
Tabla 4. Residuales en aceleración, velocidad y desplazamiento para el modelo con dos y tres SMGA para las estaciones MANZ, CJIG, MOIG, ZAIG Y ZIIG.

\begin{tabular}{|c|c|c|c|c|c|c|c|}
\hline \multirow{2}{*}{ Est. } & \multirow{2}{*}{ Componente } & \multicolumn{3}{|c|}{ Dos SMGA } & \multicolumn{3}{|c|}{ Tres SMGA } \\
\hline & & Acel. & Vel. & Desp. & Acel. & Vel. & Desp. \\
\hline \multirow{5}{*}{ 忞 } & NS & 2.23 & 1.23 & 3.02 & 1.68 & 1.17 & 1.67 \\
\hline & WE & 1.21 & 10.75 & 0.61 & 1.59 & 1.06 & 1.08 \\
\hline & Vertical & 2.70 & 5.34 & 2.00 & 1.53 & 1.14 & 1.84 \\
\hline & $\sum$ & 6.13 & 17.32 & 5.63 & 4.79 & 3.36 & 4.59 \\
\hline & NS & 0.68 & 0.59 & 0.39 & 1.84 & 1.58 & 1.60 \\
\hline \multirow{4}{*}{$\underset{\circlearrowright}{\circlearrowright}$} & WE & 1.61 & 1.56 & 0.97 & 2.29 & 1.54 & 1.55 \\
\hline & Vertical & 1.79 & 1.21 & 1.74 & 1.93 & 1.56 & 1.32 \\
\hline & $\sum$ & 4.09 & 3.35 & 3.11 & 6.05 & 4.68 & 4.47 \\
\hline & $\mathrm{NS}$ & 5.34 & 1.68 & 3.62 & 1.64 & 1.47 & 1.06 \\
\hline \multirow{4}{*}{$\frac{\mathfrak{O}}{\stackrel{0}{2}}$} & WE & 35.37 & 9.16 & 5.41 & 1.58 & 1.53 & 1.53 \\
\hline & Vertical & 54.51 & 16.48 & 8.55 & 1.49 & 1.32 & 1.26 \\
\hline & $\sum$ & 95.22 & 27.33 & 17.59 & 4.71 & 4.32 & 3.84 \\
\hline & $\mathrm{NS}$ & 29.21 & 27.93 & 27.58 & 1.33 & 1.15 & 1.20 \\
\hline \multirow{4}{*}{$\underset{N}{\stackrel{N}{\mathbb{N}}}$} & WE & 7.58 & 3.58 & 3.73 & 1.59 & 1.53 & 1.65 \\
\hline & Vertical & 7.46 & 4.58 & 4.48 & 1.58 & 1.39 & 1.42 \\
\hline & $\sum$ & 44.25 & 36.08 & 35.78 & 4.50 & 4.07 & 4.27 \\
\hline & $\mathrm{NS}$ & 21.94 & 13.94 & 6.80 & 2.15 & 1.79 & 1.17 \\
\hline \multirow{5}{*}{$\stackrel{\circlearrowright}{\Xi}$} & WE & 25.52 & 17.06 & 3.12 & 1.96 & 1.69 & 1.55 \\
\hline & Vertical & 4.97 & 1.41 & 0.87 & 1.96 & 1.48 & 1.11 \\
\hline & $\sum$ & 52.43 & 32.42 & 10.80 & 6.06 & 4.95 & 3.82 \\
\hline & TOTAL & 195.99 & 99.18 & 67.28 & 21.32 & 18.02 & 16.40 \\
\hline & $*$ & 49.00 & 24.79 & 16.82 & 5.33 & 4.51 & 4.10 \\
\hline
\end{tabular}

*Valores de residual promedio para las 5 estaciones.

Las figuras 7, 8, 9, 10, y 11 muestran la comparación entre los registros observados (rojo) y sintéticos (azul) para las tres componentes, en aceleración, velocidad y desplazamiento, de las estaciones MANZ, CJIG, MOIG, ZAIG y ZIIG, respectivamente.

Las relaciones de Somerville et al. (2002) que escalan el momento sísmico con algunos parámetros de la fuente, son comparadas con los siguientes resultados obtenidos en este estudio: tiempo de ascenso (figura 12a), área total de las SMGAs (figura 12b), área de la SMGA más grande (figura 12c), radio de la SMGA más grande (figura 12d) y distancia hipocentral a la aspereza más cercana (figura 12e). El resultado de estas comparaciones muestra que la relación entre el Mo y la distancia hipocentral a la aspereza más cercana ajusta medianamente bien, lo que no sucede para el resto de las relaciones.

\section{DISCUSIÓN}

El mejor modelo obtenido se compone de tres SMGA. El proceso de búsqueda del mejor ajuste mencionado anteriormente, y que generó 4 modelos (SMGA A, SMGA B, 2 SMGA Y 3 SMGA), muestra claramente que los valores de residual para la estación local (MANZ) disminuyeron de manera progresiva (tabla 5) al modelar con una sola SMGA y variar su localización de la posición A (residual de aceleración $=108.75)$ a la posición B (residual en aceleración $=23.00)$. Los residuales mejoraron notablemente al modelar con dos SMGA (residual de aceleración $=6.13$ ) en las localizaciones mostradas en la figura 3c. Sin embargo, el mayor ajuste se obtuvo al modelar con 3 SMGA, en las localizaciones mostradas en la figura 3d (residual de aceleración $=4.79$ ). Las componentes de velocidad y desplazamiento tienen un comportamiento similar. De igual manera para 
las estaciones regionales (CJIG, MOIG, ZAIG y ZIIG) el ajuste mejora notablemente al pasar del modelo con dos SMGA (residual en aceleración promedio $=49.00$ ) al modelo con tres SMGA (residual de aceleración promedio $=5.33$ ).

\section{3/22/01 02:06:34.5 MANZ (3 SGMA)}
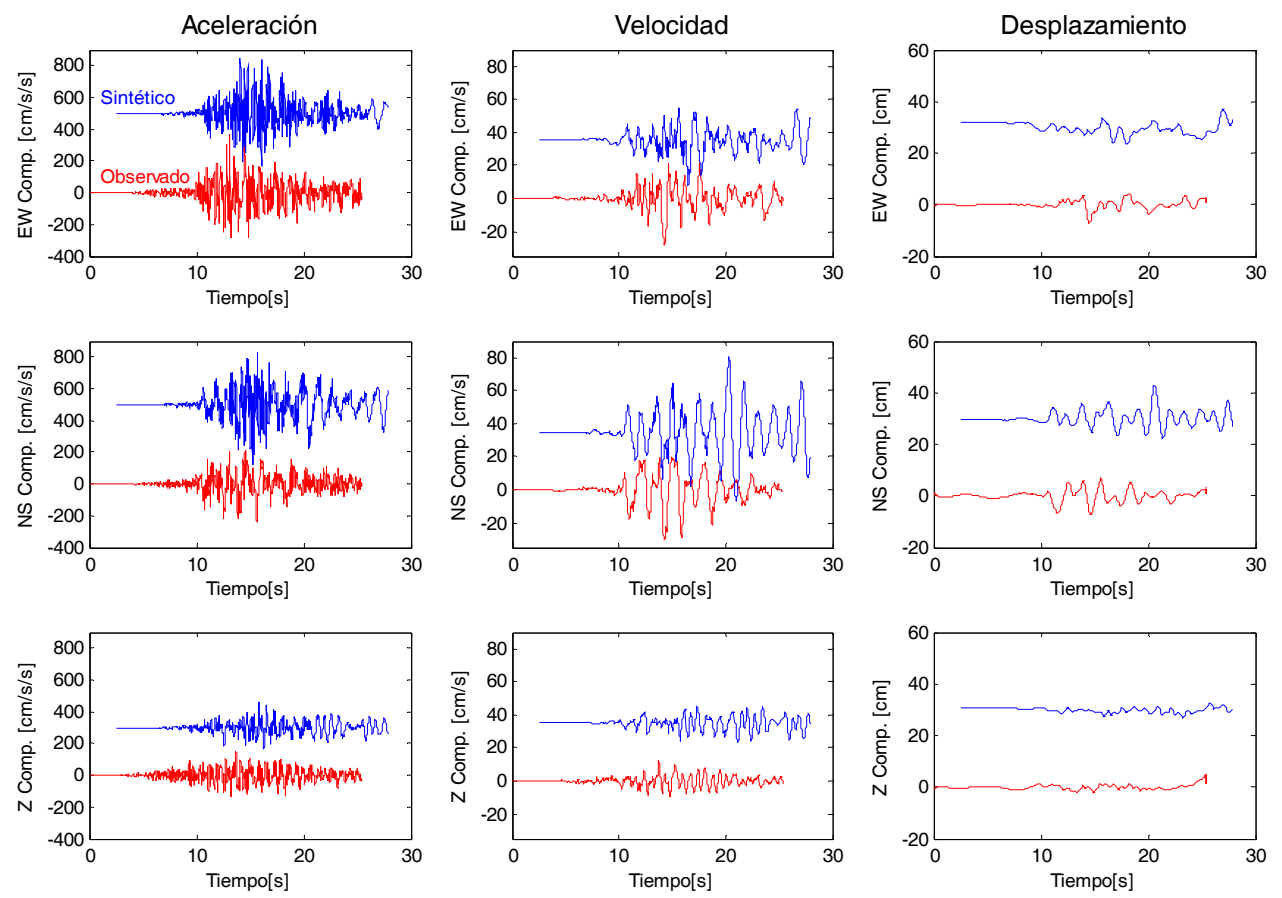

Figura 7. Simulación para la estación MANZ usando el modelo con tres SMGA.

\section{3/22/01 02:06:34.5 CJIG (3 SGMA)}
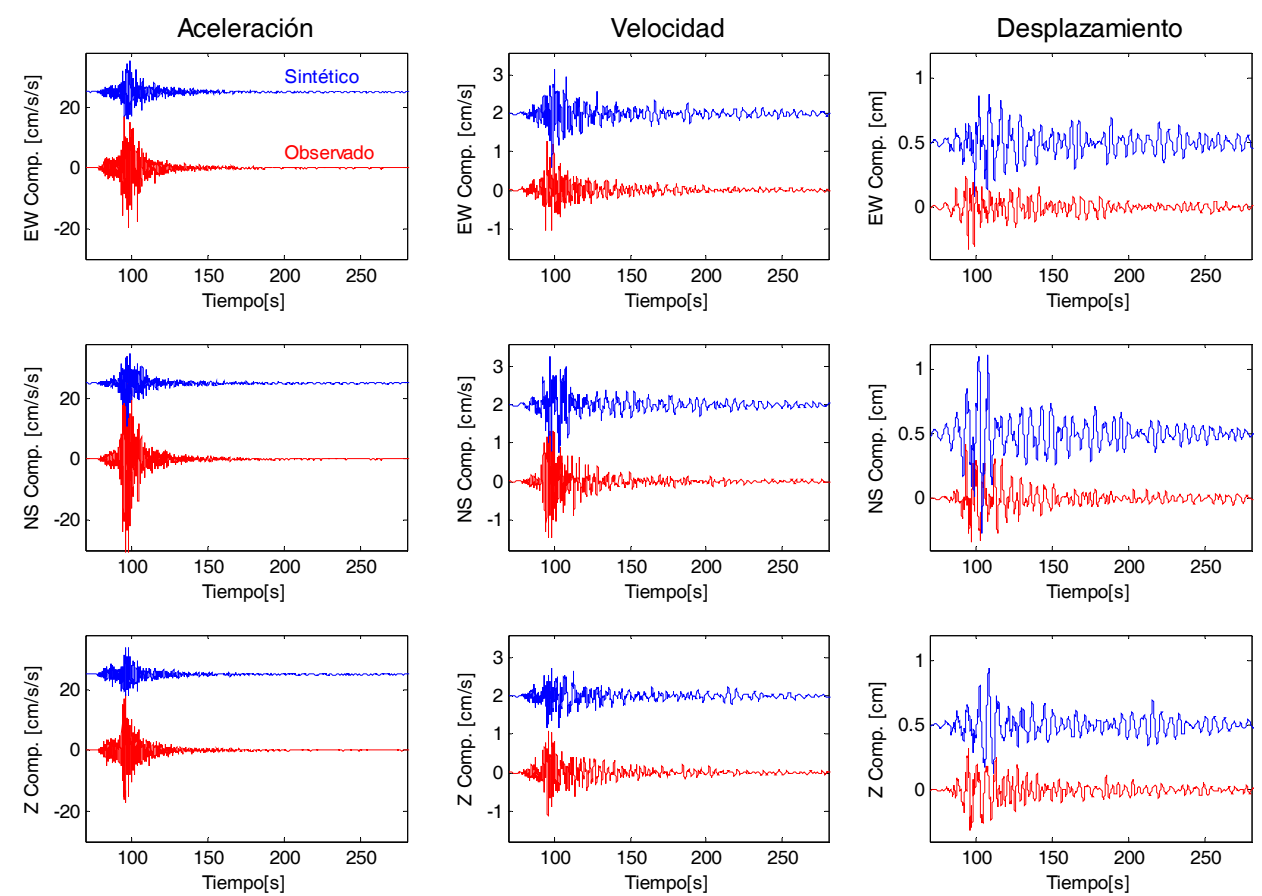

Figura 8. Simulación para la estación CJIG usando el modelo con tres SMGA. 
2003/22/01 02:06:34.5 MOIG (3 SGMA)
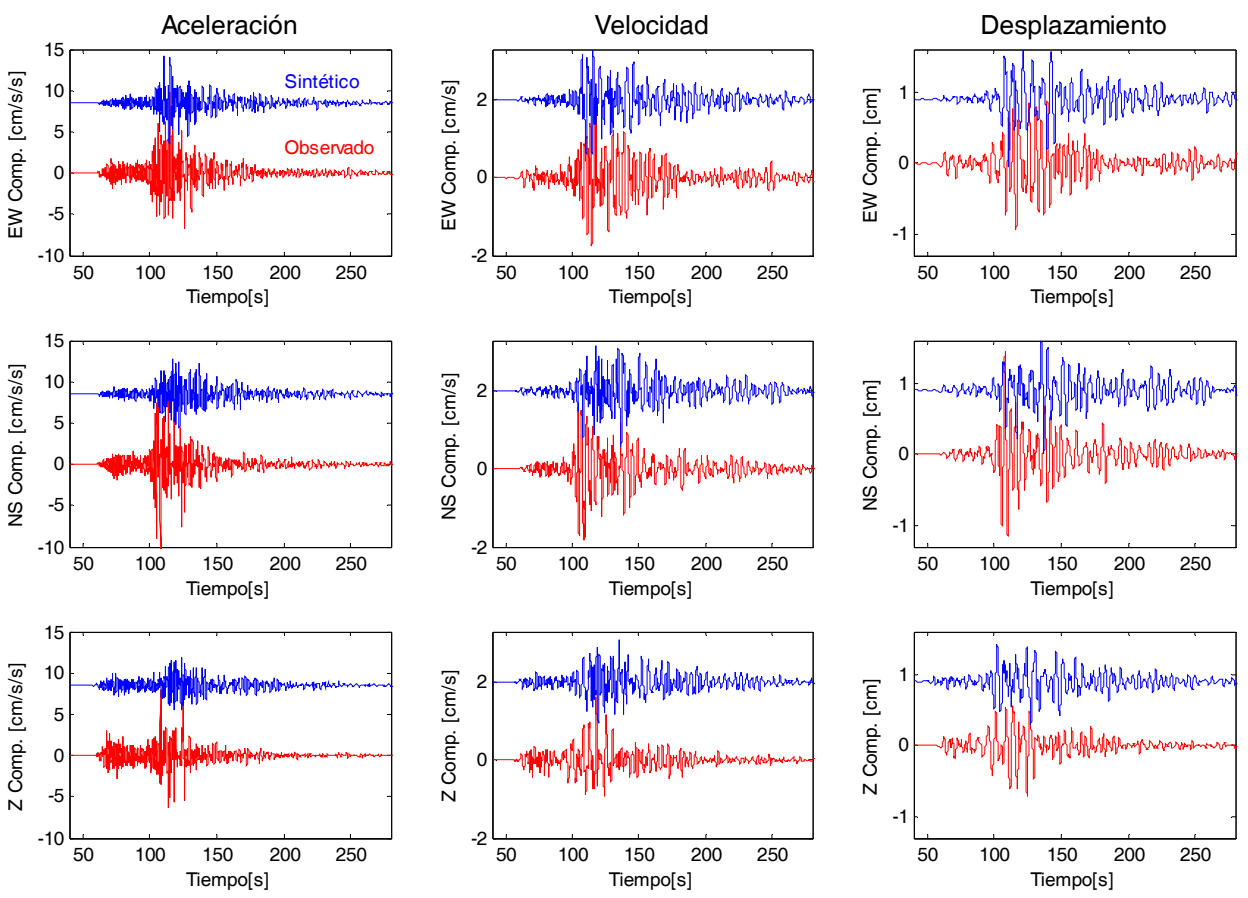

Figura 9. Simulación para la estación MOIG usando el modelo con tres SMGA.

\section{3/22/01 02:06:34.5 ZAIG (3 SGMA)}
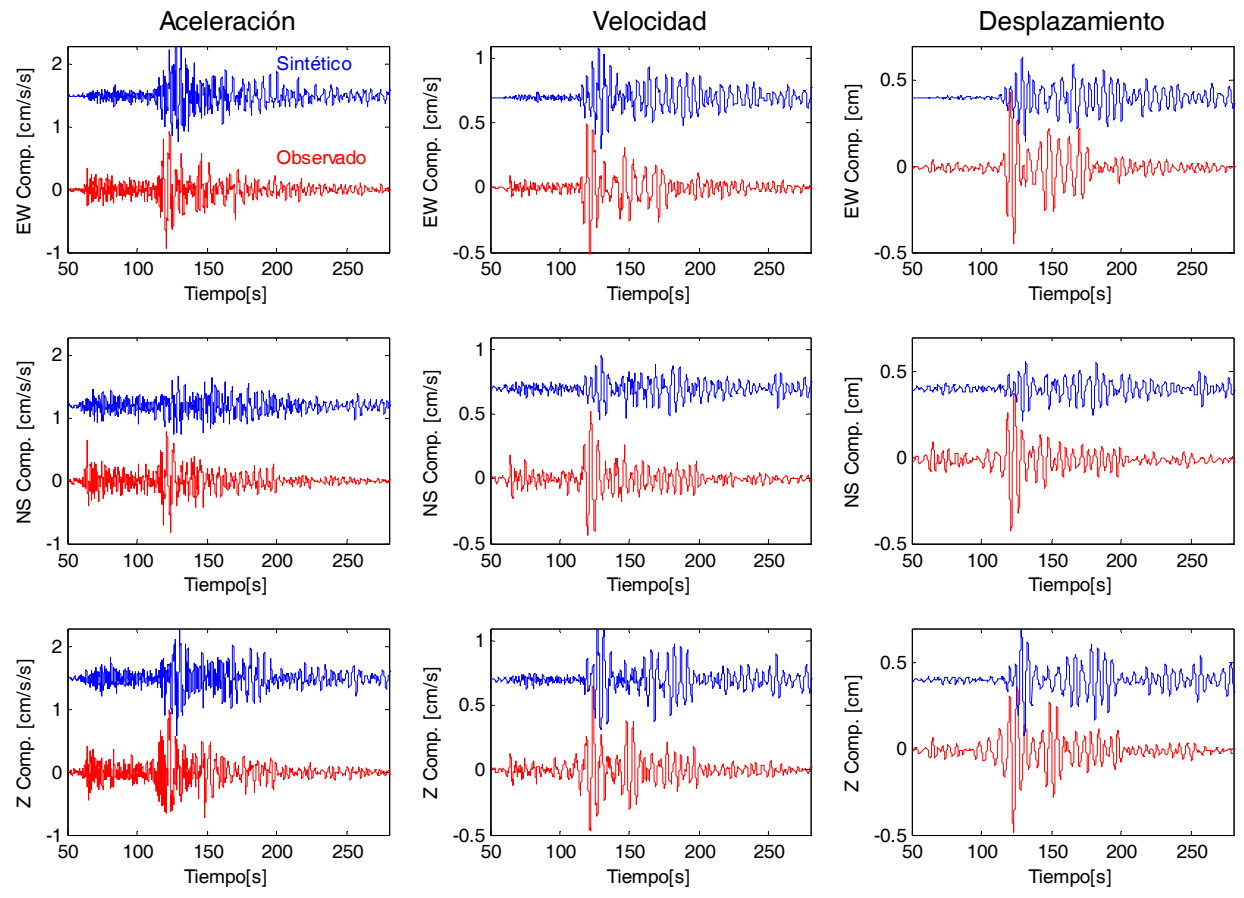

Figura 10. Simulación para la estación ZAIG usando el modelo con tres SMGA. 
2003/22/01 02:06:34.5 ZIIG (3 SGMA)
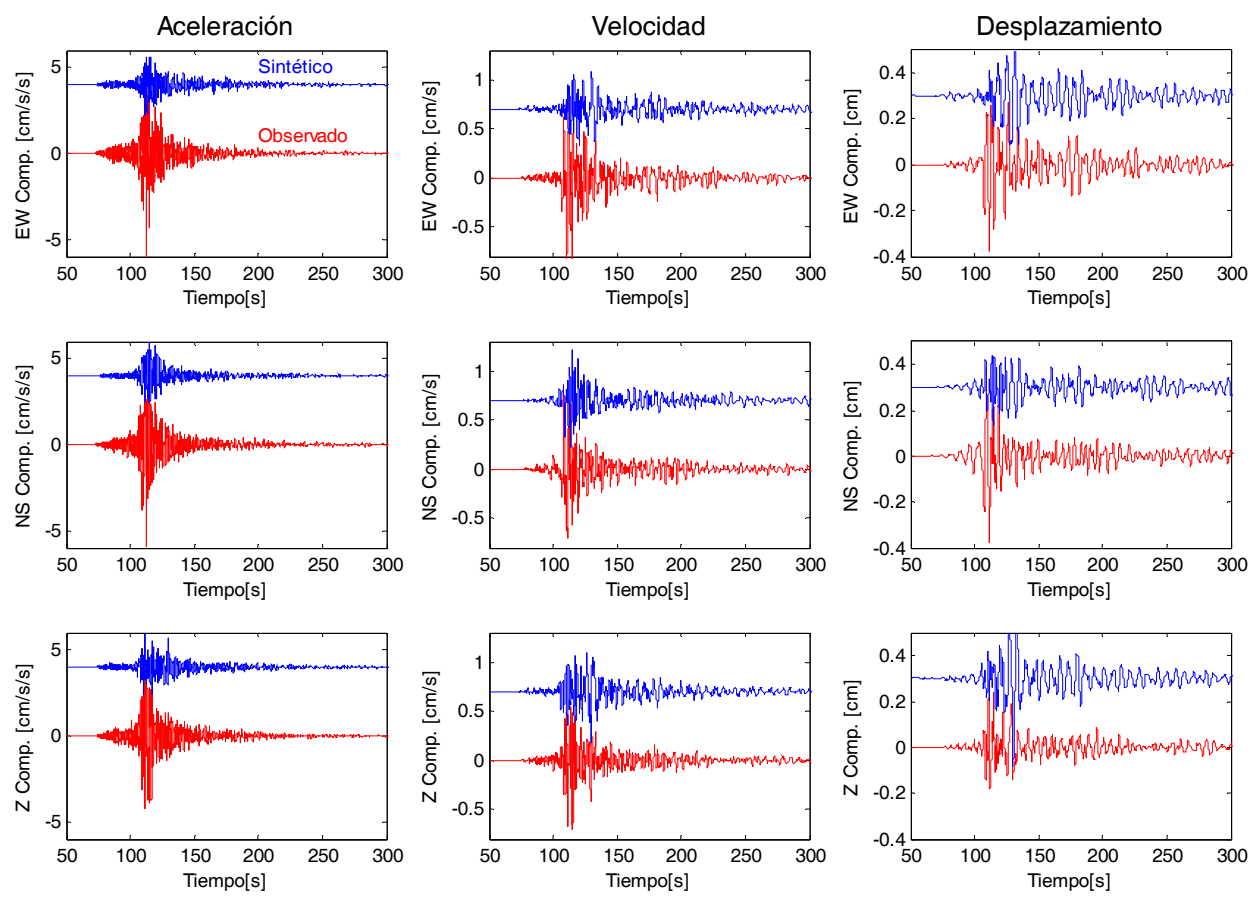

Figura 11. Simulación para la estación ZIIG usando el modelo con tres SMGA.

Este modelo de 3 SMGA, además de haber presentado los mejores ajustes en residual, guarda un gran parecido con el modelo de dislocación encontrado por Yagi et al. (2004) (Figura 13), quien reporta que el proceso de ruptura se divide en tres etapas: una primera etapa en que inicia la ruptura cerca del hipocentro; una segunda etapa en que ésta se propaga hacia el sureste y rompe la aspereza A, a $15 \mathrm{~km}$ del inicio de la ruptura, y al mismo tiempo una tercera etapa en que la ruptura se propaga al noreste y rompe la aspereza B, a $25 \mathrm{~km}$ del inicio de la ruptura. La SMGA1 encontrada en el modelo propuesto para este caso se localizó a $1.72 \mathrm{~km}$ de la aspereza A; la SMGA 2 se localiza a $5.16 \mathrm{~km}$ del hipocentro y la SMGA 3 se localiza en el mismo sitio que la aspereza B encontrada por Yagi et al. (2004).

Tabla 5. Sumatoria de residuales para las tres componentes (EW, NS y Z) en aceleración, velocidad y desplazamiento.

\begin{tabular}{crrr}
\hline Modelos para la estación & \multicolumn{3}{c}{ Residual } \\
MANZ & \multicolumn{1}{c}{ Acel. } & \multicolumn{1}{c}{ Vel. } & Desp. \\
\hline 1 SMGA (SMGA A) & 108.75 & 29.62 & 9.55 \\
1 SMGA (SMGA B) & 23.00 & 12.50 & 7.05 \\
2 SMGA & 6.13 & 17.32 & 5.63 \\
3 SMGA & 4.79 & 3.36 & 4.59 \\
Modelos para las estaciones regionales & * Residual promedio \\
CJIG, MOIG, ZAIG y ZIIG & Acel. & Vel. & Desp. \\
2 SMGA & 49.00 & 24.79 & 16.82 \\
3 SMGA & 5.33 & 4.51 & 4.10 \\
\hline
\end{tabular}

*Valores de residual promedio para las 5 estaciones.

Es notorio que la mejoría que se observa con la reducción de los residuales, lo es en general tanto en aceleración, velocidad y desplazamiento. Esto muestra la conveniencia de utilizar la ecuación 
11 para cuantificar el ajuste de los sintéticos con los observados, incluso en los registros de aceleración que contienen altas frecuencias.

Los valores de residual para las tres componentes en aceleración, velocidad y desplazamiento mostrados en la tabla 5, muestran que para la estación CJIG los residuales son menores en el modelo con dos SMGA que con el modelo con tres SMGA. Estos valores de residual no parecen corresponder con la tendencia mostrada para el resto de las estaciones. Lo anterior parece indicar que la estación CJIG no es tan sensible al modelado con la tercera SMGA como el resto de las estaciones. Sin embargo es importante notar que mientras para esta estación (CJIG), el residual aumenta solo un 0.71 $\%$ del modelo con dos SMGA al modelo con tres SMGA, para el resto de las estaciones el residual disminuye en un promedio de hasta $800 \%$ del modelo con dos SMGA al modelo con tres SMGA. El ligero aumento del residual en la estación CJIG al modelar con tres SMGA no tiene un peso significativo en el residual global obtenido.
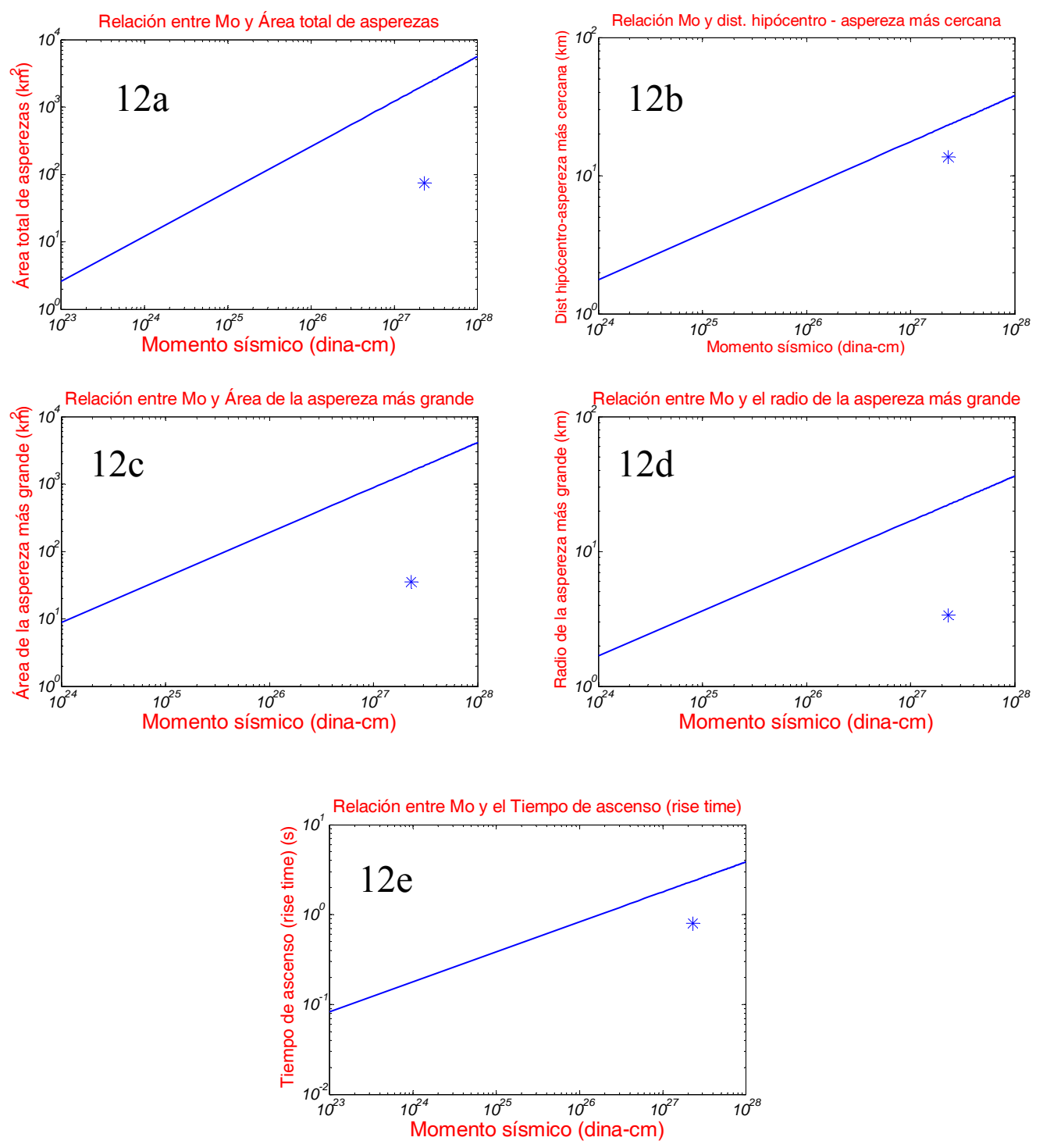

Figura 12. Comparación de los resultados obtenidos en este estudio con las relaciones de Somerville et al. (2002). 
La tabla 6 muestra la comparación de los máximos valores en aceleración, velocidad y desplazamiento para tres componentes en las cinco estaciones. Puede observarse que las estaciones MANZ y ZAIG tienen ajustes mayores al $90 \%$ en aceleración en las componentes este-oeste y vertical; en velocidad, ajustes mayores al $70 \%$ para las mismas componentes. Los ajustes en aceleración, velocidad y desplazamiento para las componentes N-S en estas mismas estaciones son menores al 60\%; de la misma manera, los ajustes en aceleración en las tres componentes para el resto de las estaciones (CJIG, MOIG y ZAIG), son menores al 60\%.

Singh et al. (2003) demuestran que la directividad de este sismo fue en dirección de la estación COIG y se propagó hacia la ciudad de Colima y al noreste del país con un acimut de $38^{\circ} \mathrm{NE}$. Las estaciones donde se dan los mejores ajustes en aceleración, velocidad y desplazamiento (estaciones MANZ y ZAIG) parecen ajustarse a la dirección de propagación de la ruptura del sismo mostrada por Singh et al. (2003). Las estaciones donde se dan ajustes menores al 50\%, estaciones CJIG, MOIG y ZIIG, parecen encontrarse fuera de esta dirección.

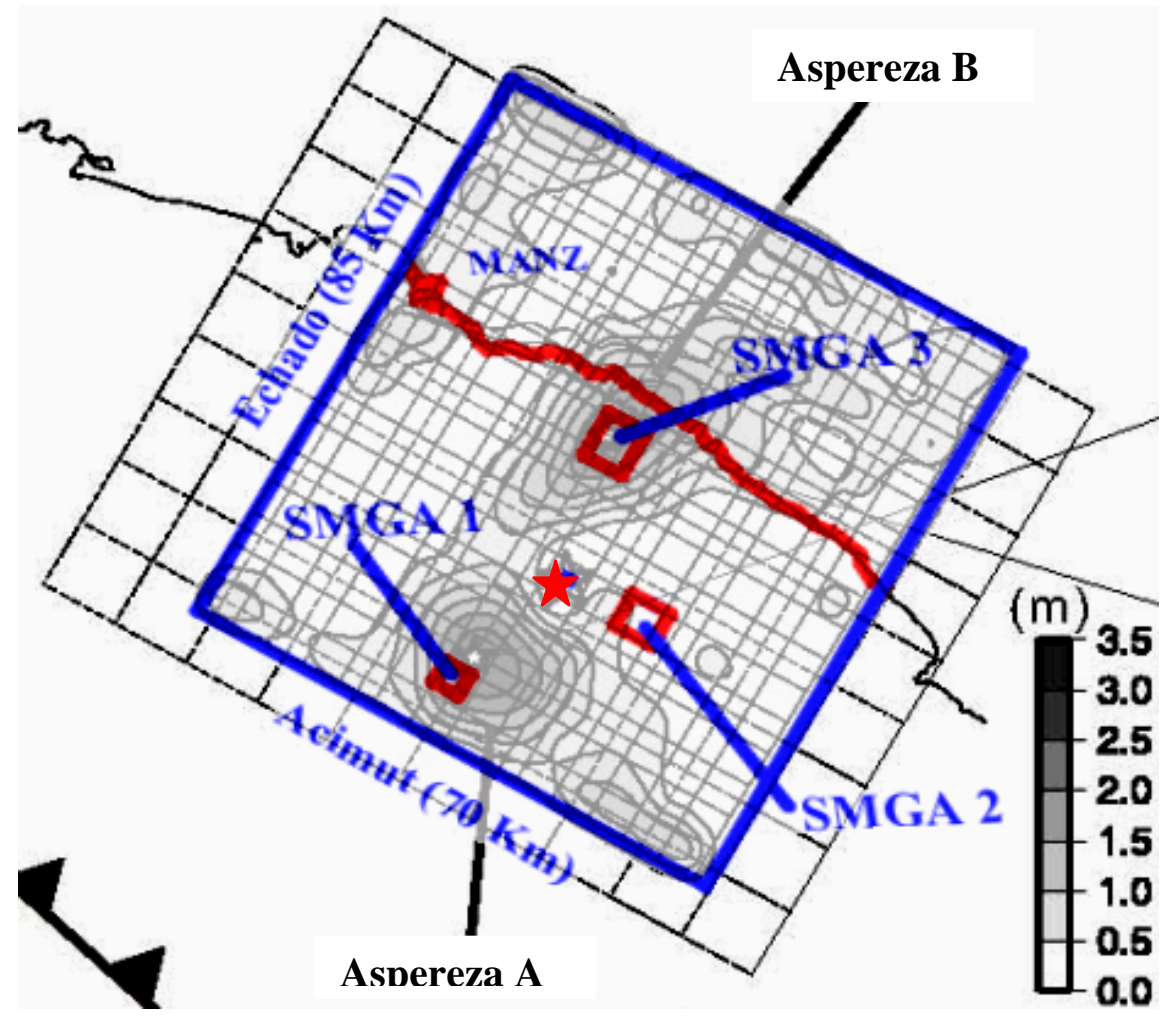

Figura 13. Comparación de las SMGAs 1, 2, y 3 que corresponden al modelo de fuente encontrado en este estudio, con las asperezas A y B del modelo de dislocación encontrado por Yagi et al. (2004) cuyos contornos aparecen en líneas tenues.

El sismo de Tecomán del 2003 se originó en el centro del graben El Gordo, propagándose en la dirección señalada con anterioridad dentro del graben de Colima. Los valores pico en aceleración, velocidad y desplazamiento para las estaciones que se encuentran fuera de la dirección de propagación del sismo (Tabla 6) pudieran ser explicados debido a un fenómeno en el que los límites del graben estén funcionando como fronteras, en donde las ondas sísmicas sufren difracción, reflexión y refracción.

Esto ocasionaría que la dirección que tomen las ondas después de llegar a estas fronteras dependa en gran medida de su trayectoria inicial, es decir de la ubicación del sismo dentro del graben. De esta manera, para poder reproducir las formas de onda en estaciones fuera del graben 
(perpendiculares a la dirección de propagación), la ubicación del sismo principal y el sismo utilizado como función de Green empíricas deberían estar lo más cerca posible. Sin embargo, la distancia que existe entre los hipocentros de estos dos sismos es de $36 \mathrm{~km}$. No obstante, no parece existir una clara relación entre las estaciones que se ubican fuera de la dirección de propagación del sismo y el ajuste de sus valores pico.

Tabla 6. Comparación para los máximos valores de velocidad, aceleración y desplazamiento (sintético y observado) en las tres componentes y para cada una de las estaciones.

\begin{tabular}{|c|c|c|c|c|c|c|c|}
\hline Comp. & Traza & Unidad & MANZ & CJIG & MOIG & ZAIG & ZIIG \\
\hline \multirow{6}{*}{3} & Aceleración Observada. & $\mathrm{cm} / \mathrm{s}^{2}$ & 372.24 & 19.49 & 9.81 & 0.94 & 6.00 \\
\hline & Aceleración Sintética. & $\mathrm{cm} / \mathrm{s}^{2}$ & 352.94 & 10.51 & 5.63 & 1.03 & 1.78 \\
\hline & Velocidad Observada. & $\mathrm{cm} / \mathrm{s}$ & 28.66 & 1.28 & 2.08 & 0.63 & 0.93 \\
\hline & Velocidad Sintética. & $\mathrm{cm} / \mathrm{s}$ & 26.69 & 1.42 & 1.40 & 0.40 & 0.39 \\
\hline & Desplazamiento Observado. & $\mathrm{cm}$ & 7.46 & 0.32 & 2.02 & 5.08 & 0.38 \\
\hline & Desplazamiento Sintético. & $\mathrm{cm}$ & 7.17 & 0.42 & 0.95 & 0.25 & 0.27 \\
\hline \multirow{6}{*}{ z } & Aceleración Observada. & $\mathrm{cm} / \mathrm{s}^{2}$ & 239.14 & 43.40 & 11.81 & 0.81 & 5.94 \\
\hline & Aceleración Sintética. & $\mathrm{cm} / \mathrm{s}^{2}$ & 375.23 & 14.25 & 4.25 & 0.47 & 2.05 \\
\hline & Velocidad Observada. & $\mathrm{cm} / \mathrm{s}$ & 30.20 & 1.48 & 1.84 & 0.53 & 0.81 \\
\hline & Velocidad Sintética. & $\mathrm{cm} / \mathrm{s}$ & 45.27 & 1.29 & 1.42 & 0.25 & 0.52 \\
\hline & Desplazamiento Observado. & $\mathrm{cm}$ & 7.15 & 0.56 & 1.45 & 9.21 & 0.39 \\
\hline & Desplazamiento Sintético. & $\mathrm{cm}$ & 12.95 & 0.77 & 0.90 & 0.19 & 0.17 \\
\hline \multirow{6}{*}{$\mathrm{N}$} & Aceleración Observada. & $\mathrm{cm} / \mathrm{s}^{2}$ & 144.55 & 18.51 & 7.98 & 1.01 & 4.22 \\
\hline & Aceleración Sintética. & $\mathrm{cm} / \mathrm{s}^{2}$ & 163.53 & 9.13 & 3.32 & 0.91 & 2.03 \\
\hline & Velocidad Observada. & $\mathrm{cm} / \mathrm{s}$ & 12.43 & 1.14 & 1.62 & 0.65 & 0.70 \\
\hline & Velocidad Sintética. & $\mathrm{cm} / \mathrm{s}$ & 11.76 & 0.82 & 1.08 & 0.43 & 0.52 \\
\hline & Desplazamiento Observado. & $\mathrm{cm}$ & 5.13 & 1.06 & 0.70 & 7.59 & 0.24 \\
\hline & Desplazamiento Sintético. & $\mathrm{cm}$ & 2.93 & 0.45 & 0.61 & 0.32 & 0.38 \\
\hline
\end{tabular}

Al aplicar las relaciones propuestas por Somerville et al. (2002) para sismos de subducción, que relacionan el momento sísmico con diversos parámetros de la fuente, así como el momento sísmico con algunas características de las SMGAs generadas en este estudio, se observa que la relación entre el Mo y la distancia hipocentral a la aspereza más cercana se ajusta en un $53 \%$ al valor propuesto por las relaciones de Somerville et al. (2002). El número de asperezas promedio en nuestro modelo es de 3, muy cercano a los 2.4 que propone Somerville et al. (2002). Sin embargo, al aplicar la relación entre el Mo y el área de ruptura propuesta por Somerville et al. (2002), el área obtenida en la inversión realizada por Yagi et al. (2004) es solo un 27\% del valor propuesto. Para esta misma relación, Rodríguez-Lozoya et al. (2007) obtiene un área del $22.40 \%$ del valor propuesto en las relaciones de Somerville et al. (2002). Garduño (2006) aplicó estas mismas relaciones al modelo que obtuvo para el sismo del 15 de julio de 1996 en las costas del estado de Guerrero, y el área de ruptura obtenida en su modelo, fue de solo 3\% del valor propuesto por Somerville et al. (2002). Para el resto de las relaciones obtenidas con el modelo que se presenta en este artículo, los valores comparativos son menores al $15 \%$ con relación a los obtenidos por Somerville et al. (2002). Los resultados ya mencionados obtenidos por Yagi et al. (2004), Rodríguez-Lozoya et al. (2007), Garduño (2006), así como los obtenidos en este artículo, podrían sugerir que estas relaciones propuestas por Somerville et al. (2002) no son todas aplicables para la zona de subducción en México.

Gran cantidad de proyectos de investigación relacionados con el peligro sísmico frecuentemente se limitan a estudiar una fracción del problema (abordando únicamente lo relacionado con la fuente sísmica, considerados aisladamente los efectos de trayecto, o bien realizando estudios puntuales de efectos de sitio que no incluyen análisis alguno de las posibles fuentes sísmicas). 
La aplicación de modelos de fuente como el desarrollado en esta investigación aporta dos elementos originales y poderosos de la sismología que permiten generar aplicaciones novedosas y muy útiles a la ingeniería sísmica en nuestro país. La primera es la metodología aplicada en esta investigación (funciones de Green empíricas) que considera de manera conjunta e implícita los diferentes factores que intervienen en el peligro sísmico, desde la fuente, trayectoria hasta los efectos de sitio. La segunda contribución es quizás la más importante aplicación de esta metodología, el modelo de fuente obtenido permitirá simular las historias de aceleración y adicionalmente los espectros de respuesta del evento de Tecomán en lugares donde no existía instrumentación en el momento del sismo. Al aplicar el modelo de fuente obtenido en esta investigación a aproximadamente 33 registros existentes del sismo del 19 de noviembre de 2006 (registrados en equipos de distintas instituciones en el interior y en las cercanías del estado de Colima) podrán simularse los acelerogramas, espectros de respuesta, PGA e $\mathrm{I}_{\mathrm{MM}}$ del sismo de Tecomán en estos sitios donde hasta el año 2003 no existía instrumentación, razón por la que no fue posible registrar dicho evento. Como ya se mencionó estos acelerogramas tendrán implícitos los efectos de la fuente, trayectoria y de sitio.

La validación de estos resultados se puede realizar al comparar las PGA obtenidas mediante métodos estadísticos contra las generadas con nuestro modelo. De la misma manera podrán validarse al comparar las intensidades ya publicadas para este sismo por diversos autores con las generadas con nuestro modelo. Las anteriores validaciones y adicionalmente el hecho de que estas simulaciones se obtuvieron al aplicar un modelo de fuente, resultado de un proceso en que los residuales fueron disminuyendo progresivamente hasta obtener el mínimo posible, permitirían alcanzar un alto grado de confiabilidad al estimar las respuestas de las construcciones del sitio.

La determinación de la cantidad y tamaño de las SMGAs son factores que influyen de manera determinante en las aceleraciones e intensidad de un sismo debido a que la mayor cantidad de energía liberada por el sismo se genera en estas SMGAs. La posición de éstas determina la directividad del sismo y la directividad puede incrementar drásticamente la intensidad esperada en algunas zonas. El establecer el número de las áreas de generación de movimientos fuertes de un sismo su tamaño y su posición así como los demás parámetros de la fuente considerados permite generar un modelo que al ser aplicado de acuerdo a la metodología mostrada en este artículo genera información muy importante para la ingeniería sísmica (historias de aceleración, PGA e $\mathrm{I}_{\mathrm{MM}}$ ) muy cercanas a la realidad.

La aplicación de metodologías para el estudio de la fuente deben de ir mas allá que el conocimiento de la propia fuente sísmica y tener aplicaciones prácticas y útiles en la ingeniería sísmica. El avance de la instrumentación en México permite que la metodología mostrada en este artículo pueda ser aplicada a otras zonas del país y así conocer parámetros útiles a la ingeniería sísmica (historias de aceleración, PGA e $\mathrm{I}_{\mathrm{MM}}$ ) de grandes sismos en sitios donde en su momento no existió instrumentación.

Tradicionalmente después de un sismo se instrumentan inmediatamente las zonas cercanas al epicentro con el fin de conocer la ubicación de las réplicas con mayor precisión. Esta visión puede ampliarse y después de un gran sismo llevar la instrumentación ahora a los puntos más críticos de las zonas urbanas, con mayor densidad de población y obras civiles de importancia. Al aplicar esta metodología a las principales réplicas, podrán simularse las historias de aceleración PGA e $\mathrm{I}_{\mathrm{MM}}$ del sismo principal en todos esos puntos críticos sin necesidad de haber tenido una instrumentación previa y por lo tanto permanente en los mismos. Los espectros de respuesta así generados pueden ser comparados con los del reglamento de construcción de la zona y determinar la validez del mismo. Para el caso de las construcciones que hayan sufrido daños podrán determinarse si éstos fueron debidos a un inadecuado reglamento de construcción o bien a serias deficiencias en la aplicación del mismo. 


\section{CONCLUSIONES}

Se generó un modelo para el sismo de Tecomán del 21 de Enero de 2003 aplicando el método de las funciones empíricas de Green. Se utilizaron para ello los registros de aceleración de dos sismos, el sismo de Tecomán como sismo principal y el sismo del 21 de noviembre de 2006 como función de Green empírica. Se utilizaron los datos de aceleración registrados en la estación MANZ, obtenidos de CIRES y los datos de velocidad de 4 estaciones regionales de banda ancha del SSN que proporcionaron buena cobertura acimutal del sismo en estudio. Se siguió un proceso en el modelado en 3 etapas que incluyó una, dos y tres SMGA para cada etapa, respectivamente. Los valores de residual disminuyeron y se ajustaron progresivamente en cada etapa. De esta manera, el mejor ajuste se obtuvo al modelar con tres SMGA. Este modelo, además de haber presentado los mejores ajustes en residual, guarda un gran parecido con el modelo de dislocación encontrado por Yagi et al. (2004). La SMGA 1, encontrada en el modelo desarrollado, se localiza a $1.72 \mathrm{~km}$ de la aspereza A reportada por Yagi et al. (2004). La SMGA 2 se localiza a $5.16 \mathrm{~km}$ del hipocentro. La SMGA 3 se localiza en el mismo sitio que la aspereza B, encontrada por Yagi et al. (2004).

La aplicación de modelos de fuente como el desarrollado en esta investigación aporta dos elementos originales y poderosos de la sismología que permiten generar aplicaciones novedosas y muy útiles a la ingeniería sísmica en nuestro país. La primera es la metodología aplicada en esta investigación (funciones de Green empíricas) que considera de manera conjunta e implícita los diferentes factores que intervienen en el peligro sísmico, desde la fuente, trayectoria hasta los efectos de sitio. La segunda contribución es quizás la más poderosa aplicación de esta metodología, el modelo de fuente obtenido permitirá simular los acelerogramas, espectros de respuesta, PGA e $\mathrm{I}_{\mathrm{MM}}$ del sismo de Tecomán en estos sitios donde hasta el año 2003 no existía instrumentación. La validación de estos resultados se puede realizar al comparar las PGA obtenidas mediante métodos estadísticos contra las generadas con nuestro modelo. De la misma manera podrán validarse al comparar las intensidades ya publicadas para este sismo por diversos autores con las generadas con nuestro modelo. Las anteriores validaciones y adicionalmente el hecho de que estas simulaciones se obtuvieron al aplicar un modelo de fuente resultado de un proceso en que los residuales fueron disminuyendo progresivamente hasta obtener el mínimo posible permitirían alcanzar un alto grado de confiabilidad al estimar las respuestas de las construcciones del sitio.

\section{RECONOCIMIENTOS}

Agradecemos el apoyo del Ing. Lucio Camarillo del Centro de Instrumentación y Registro Sísmico (CIRES) por proporcionar los registros de aceleración de la estación Manzanillo; del Dr. Carlos Valdés González y la MC. Caridad Cárdenas, por proporcionar los registros de la red de banda ancha del Servicio Sismológico Nacional. Agradecemos al Consejo Nacional de Ciencia y Tecnología (CONACYT) por el apoyo proporcionado a este proyecto (Proyecto SEP-2003-C02-43880/A). Agradecemos ampliamente a los dos revisores anónimos, por sus invaluables observaciones y comentarios.

\section{REFERENCIAS}

Aguirre, J, K Irikura y K Kudo (1994), "Estimation of strong ground motions on hard rock and soft sediments sites in the Ashigara Valley using the empirical Green's function method", Bulletin Disaster Prevention Research Institute, Kyoto University, Vol. 44, part 1, No. 379, pp. 45-64.

Bandy, W, C Mortera, J Urrutia y T Hilde (1995), "The subducted Rivera-Cocos plate boundary: where is it, what is it, and is its relationship to the Colima rift?", Geophysical Research Letters, Vol. 22, pp. 3075-3078. 
Garduño, N (2006), "Proceso de la fuente del sismo del 15 de julio de 1996 usando el método de la función de Green empírica y algoritmos genéticos", Tesis de Licenciatura, Facultad de Ingeniería, Universidad Nacional Autónoma de México, págs. 111.

Irikura, K (1986), "Prediction of strong accelerations motions using empirical Green's function", Proceedings of the Japan Earthquake Engineering Symposium 11th, pp. 151156 (en Japonés).

Kamae, K y K Irikura (1998), "Source model of the 1995 Hyogo-Ken Nanbu earthquake and simulation of near source ground motion", Bulletin of the Seismological Society of America, Vol. 88, pp. 400-412.

Miyake, H, T Iwata y K Irikura (2003), "Source characterization for broadband ground motion simulation: kinematic heterogeneous source model and strong motion generation area", Bulletin of the Seismological Society of America, Vol. 93, pp. 2531-2545.

Nozu, N, K Irikura (2008), "Strong motion generation area of great subduction-zone earthquake waveform inversion with empirical Green's function for the 2003 Tokachi-Oki earthquake", Bulletin of the Seismological Society of America, Vol. 98, pp. 180-197.

Rodríguez-Lozoya, H, L Quintanar, C Rebollar, J Gómez, Y Yagi, T Domínguez, G Reyes, C Javier, y L Alcántara (2007), "Source characteristics of the 22 January $2003 \mathrm{M}_{\mathrm{w}} 7.5$ Tecomán, México, earthquake and its rupture process", enviado para su publicación a Journal of Geophysical Research.

Singh, K, J Pacheco, L Alcántara, G Reyes, M Ordaz, A Iglesias, M Alcocer, C Gutiérrez, C Valdez, V Kostoglodov, C Reyes, T Mikumo, R Quaas, R Anderson y contribuciones de otros autores (2003), "A preliminary report on the Tecomán, México earthquake of 22 January $2003\left(M_{w} 7.4\right)$ and its effects", Seismological Research Letters, Vol. 74, No. 3, pp. 279-289.

Somerville, P, K Irikura, R Graves, S Sawada, D Wald, N Abrahamson, Y Iwasaki, T Kagawa, N Smith y A Kowada (1999), "Characterizing crustal earthquake slip models for the prediction of strong motion", Seismological Research Letters, Vol. 70, pp. 59-80.

Somerville, P, T Sato, I Toru, N Collins, K Dan y F Hiroyuki (2002), "Characterizing subduction earthquake slip models for the prediction of strong motion", Proceedings of the Japan Earthquake Engineering Symposium 11th, pp. 163-166 (en Japonés).

Yagi, Y, T Mikumo, J Pacheco y G Reyes (2004), "Source rupture process of Tecomán, Colima, México earthquake of January 22, 2003, determined by joint inversion of teleseismic bodywave and near-source data”, Bulletin of the Seismological Society of America, Vol. 94, No. 5, pp. 1795-1807. 